\title{
ADNKA overcomes SARS-CoV2-mediated NK cell inhibition through non-spike antibodies
}

Fielding $\mathrm{CA}^{1}$, Sabberwal $\mathrm{P}^{1}$, Williamson $\mathrm{JC}^{2}$, Greenwood EJD², Crozier TWM ${ }^{2}$, Zelek $\mathrm{W}^{1}$, Seow $\mathrm{J}^{3}$, Graham $\mathrm{C}^{3}$, Huettner $\mathrm{I}^{3}$, Edgeworth $\mathrm{JD}^{3,4}$, Morgan $\mathrm{BP}^{1}$, Ladell $\mathrm{K}^{1}$, Eberl $\mathrm{M}^{1}$, Humphreys $\mathrm{IR}^{1}$, Merrick $\mathrm{B}^{3,4}$, Doores $\mathrm{K}^{3}$, Wilson $\mathrm{SJ}^{5}$, Lehner $\mathrm{PJ}^{2}$, Wang $\mathrm{ECY}^{1}$, Stanton $\mathrm{RJ}^{1 *}$

1. Division of Infection and Immunity, School of Medicine, Cardiff University, CF14 4XN

2. Cambridge Institute for Therapeutic Immunology \& Infectious Disease, Jeffrey Cheah Biomedical Centre, Cambridge Biomedical Campus, University of Cambridge, Cambridge CB2 OAW, UK

3. Department of Infectious Diseases, School of Immunology \& Microbial Sciences, King's

College London, London, UK

4. Department of Infectious Diseases, Guy's and St Thomas' NHS Foundation Trust, London

5. MRC - University of Glasgow Centre for Virus Research, 464 Bearsden Road, Glasgow, G61 $1 \mathrm{QH}$

*Corresponding author, StantonRJ@cardiff.ac.uk 


\begin{abstract}
SARS-CoV-2 antagonises the cellular interferon response, but whether the virus manipulates cellular immunity is unclear. An unbiased proteomic approach to determine how cell surface protein expression is altered on SARS-CoV-2-infected lung epithelial cells showed downregulation of activating NK cell ligands: B7-H6, MICA, ULBP2, and Nectin1, but no effect on surface MHC-I expression. NK ligand downregulation correlated with a reduction in NK cell activation by infected cells, and was overcome by antibody-dependent NK cell activation (ADNKA). Depletion of spike-specific antibodies confirmed their dominant role in virus neutralisation, but these antibodies played only a minor role in ADNKA compared to antibodies to other viral proteins, including ORF3a, Membrane, and Nucleocapsid. In contrast, ADNKA induced following vaccination was focussed solely on spike, was weaker than ADNKA following natural infection, and was not boosted by the second dose. These insights have important implications for understanding disease progression, vaccine efficacy, and vaccine design.
\end{abstract}




\section{Introduction}

Both innate and adaptive immunity are critical for control of viral infection. As a result, the capacity for many pathogens to cause disease is dependent on their ability to antagonise immune pathways, with the most successful pathogens carrying a vast array of genes capable of inhibiting all arms of the immune system ${ }^{1,2}$. In the case of SARS-CoV2, the virus antagonises interferon induction via the activity of at least 10 genes $^{3-6}$. However, whether SARS-CoV2, or indeed any coronavirus, is capable of counteracting other arms of immunity is currently unknown.

Many successful viruses interfere with cellular immunity; for example, downregulation of MHC-I inhibits CD8+ T-cell mediated cytotoxicity ${ }^{2}$. The cellular immune system counters this through Natural Killer (NK) cells, which display a number of dominant inhibitory receptors to MHC-I, and are therefore more likely to activate in its absence. However, NK cell activation also requires activation signals from a wide range of activating receptors ${ }^{7}$. The ligands for these can be induced on the surface of target cells in response to stress, infection, or transformation. For example, the stress ligands MICA, MICB, and ULBP1-6, all bind to the ubiquitously expressed activating receptor NKG2D. To limit NK cell activation, some viruses have therefore evolved to also manipulate levels of these NK activating ligands, reducing NKcell mediated control ${ }^{1}$. Whether similar manipulations underlie the ability of SARS-CoV2 to establish infection and cause disease in humans has not been investigated to date.

NK cells intrinsically function early in infection, limiting pathogen spread prior to the induction of adaptive immunity, but can also operate within the adaptive phase via antibody dependent NK activation (ADNKA) which leads to antibody dependent cellular cytotoxicity (ADCC). Here, antibodies bind to viral antigens expressed on the infected cell surface, and activate NK cells through binding of Fc to the activating receptor CD16 on the NK cell. This leads to release of cytotoxic granules, and the production of TNF $\alpha$ and IFN $\gamma$, contributing significantly to a proinflammatory environment. Multiple lines of evidence indicate that ADCC is an important function in COVID-19; ADCC is strongly induced following infection with SARS-CoV2 ${ }^{8-11}$, and delayed induction of ADCC correlates with severe disease ${ }^{12}$. Furthermore, Fc mediated functions correlate with protection in Rhesus models of vaccination ${ }^{13}$, and are induced by vaccination in humans ${ }^{14,15}$. Many neutralising antibodies targeting the SARS-COV2 spike 
protein also demonstrate an ability to activate $A D C C^{16-18}$, and the ability of these antibodies to control SARS-CoV2 infection in animal models is enhanced by Fc-dependent mechanisms of action ${ }^{19-22}$. Finally, 'adaptive' NK cells, marked by expression of NKG2C, CD57, and LIR-1 ${ }^{23}$, are exceptionally potent ADCC-mediators, and expansion of these cells has been observed in severe COVID-1924, while genetic loss of NKG2C is a risk factor for severe disease ${ }^{25}$.

Taken together, ADCC is likely to play an important role in COVID-19. However significant limitations remain in our understanding of this process. Most importantly, studies to date have focussed on spike protein as the dominant ADCC target, using either plate-bound protein or transfected cells $s^{8-12,14-18}$. While viral entry glycoproteins are often found on the infected cell surface and can mediate ADCC when expressed alone, other viral proteins may be the principle mediators of ADCC during infection. Indeed, we have previously shown, in the context of other viruses, that non-structural proteins mediate more potent ADCC responses than entry glycoproteins ${ }^{26}$. It is therefore important to evaluate responses in the context of productive viral infection.

To address these critical gaps in our understanding, we used a proteomic approach to quantitate the changes in cell surface protein expression of a lung epithelial cell line, that is permissive to efficient SARS-CoV2 infection ${ }^{27}$. We found that SARS-CoV2-infected cells show downregulation of multiple activating NK ligands, resulting in a reduced ability of NK cells to respond to infected cells, with no effect on cell surface MHC-I. Following natural infection, NK inhibition was overcome through ADNKA. However this was not primarily driven by spike antibody, but dominated by antibody to other viral proteins such as nucleocapsid, membrane and ORF3a. Critically, spike was not only a poor ADNKA target following natural infection, but was also a poor activator of ADNKA following vaccination. Thus, the breadth of ADNKA responses is limited in current vaccination strategies. The addition of antigens such as nucleocapsid would recruit additional effector mechanisms, and may reduce reduction in vaccine efficacy following mutation of spike in naturally circulating virus variants. 


\section{$\underline{\text { Results }}$}

\section{SARS-CoV-2 remodels the Plasma Membrane Proteome}

Immune cells recognise and interact with infected cells through cell surface ligands. We therefore wanted to gain a comprehensive and unbiased overview of how SARS-CoV-2 infection changes the plasma membrane protein landscape. We performed plasma membrane enrichment through selective aminooxy-biotinylation (Plasma Membrane Profiling; PMP), a methodology that we previously developed ${ }^{28}$ and have used to interrogate infection by $\mathrm{HIV}^{29}$, HCMV $26,30-33$, and HSV 34 .

High infection rates are critical to minimise confounding effects from bystander cells. We therefore applied PMP to SARS-CoV-2 infected A549-ACE2-TMPRSS2 (AAT) cells, which are highly permissive to SARS-CoV- $2^{27}$ and infection at a high multiplicity of infection (MOI) results in over $90 \%$ infection by $24 \mathrm{~h}$ (Fig. $1 \mathrm{~A}$,). We utilised TMT based quantification to compare PM protein abundance in uninfected cells, and cells infected for 12, 24, and $48 \mathrm{~h}$ (Fig 1B). In total, 4953 proteins were quantitated, including 2227 proteins with gene ontology annotation associated with the cell surface and plasma membrane (Fig S1A). These proteins made up nearly $80 \%$ of the total protein abundance, indicating a high degree of enrichment for plasma membrane proteins, comparable with our previous PMP datasets ${ }^{28,29}$.

Five SARS-CoV-2 viral proteins were detected, including spike, ORF3A, and membrane, which have previously been annotated as having a cell surface localisation (Fig. 1C). Consistent with prior reports, the viral receptor ACE2 was strongly downregulated after infection ${ }^{35}$, while TMPRSS2 expression was unaffected (Fig. 1C). Overall, of the 2227 PM proteins quantified with more than one peptide, 914 showed changes which were both significant and with a greater than 1.5-fold over the course of the experiment. Clustering of proteins by their temporal pattern of change has previously shown utility in defining classes of regulated proteins and predicting underlying mechanisms ${ }^{29,36,37}$. Here, we used K-means clustering to define 5 groups of significantly altered proteins by their temporal profile.

To assess the innate immune response triggered by SARS-CoV-2 infection, the clusters were interrogated for the presence of a predefined list of interferon alpha-inducible genes. Thirteen of the 23 detected PM genes associated with the interferon response fell into cluster 
5 (Fig S1B), which describes proteins with an increase in expression at $48 \mathrm{~h}$, examples shown in Fig S1C. As the SARS-CoV-2 viral replication cycle is completed in less than $24 \mathrm{~h}^{6}, \mathrm{SARS}-\mathrm{CoV}$ 2 is able to evade innate immune recognition and/or supress IFN signalling until long after virus egress has been achieved.

To identify potential innate and adaptive immune evasion strategies employed by SARS-CoV2, we focused on cluster 1 , which describes proteins downregulated from early in the time course. This cluster was subject to gene ontology and pathway analysis to define proteins related by functional and structural similarities (Fig 2). Several classifications enriched in this cluster are particularly relevant, including the downregulation of several cytokine receptors, including IFNAR1. Much of the SARS-CoV-2 genome is dedicated to antagonising a type I interferon response ${ }^{6}$ and the plasma membrane downregulation of IFNAR1 presents a previously undescribed strategy to prevent exogenous signalling.

Also of note is the downregulation of heparan sulfate proteoglycans, the syndecan (SDC), glypican (GPC) and neurophillin (NRP) proteins, which together encompass the families of heparan sulfate modified proteins expressed on epithelial cells ${ }^{38}$. NRP1 is a cell entry factor for SARS-CoV-2 ${ }^{39,40}$, and SARS-CoV-2 spike is also reported to bind heparan sulphated proteins ${ }^{41}$. The decrease in cell surface expression of heparan sulfated proteins may therefore enable the egress of viral particles and prevent super-infection, and is reminiscent of the capacity of other viruses to downregulate both primary viral receptors and co-receptors ${ }^{42}$.

\section{SARS-CoV2 modulates multiple NK cell ligands, and inhibits NK cell activation}

Our experience with PMP has emphasised the modulation of NK ligands as an important immune evasion mechanism ${ }^{30,37}$. As these ligands are poorly annotated in public databases, we investigated the PMP dataset for previously described ligands ${ }^{7}$, of which 18 were detected (Fig S2). MHC-I proteins HLA-B to HLA-E were upregulated in cluster 5, likely reflecting a late IFN-mediated upregulation, while HLA-A was unchanged. By contrast, the activating NK-cell ligands MICA, ULBP2, Nectin1, and B7-H6 (NCR3LG1) were significantly downregulated in cluster 1 , and was confirmed by flow cytometry (Fig 3A). To determine how these changes affected NK cell activation, we incubated SARS-CoV2 infected cells with PBMC, and measured (i) degranulation of $\mathrm{CD}^{-} \mathrm{CD}^{2} 6^{+} \mathrm{NK}$ cells (Fig 3B), (ii) production of IFN $\gamma$ (Fig 3C) and (iii) TNF $\alpha$ (Fig 3D). Consistent with data using other viruses ${ }^{26}$, CD107a was activated on a higher 
proportion of cells than IFN $\gamma$ and TNF $\alpha$. Nevertheless, all three markers of NK activation were significantly reduced in SARS-CoV2 infected versus mock infected cells, suggesting that the virus has evolved to inhibit NK cell responses. Interestingly, all 4 ligands have orthologues in bats (the likely reservoir species for most coronaviruses), with one ligand (Nectin1) showing extremely high identity (>96\%) to the human gene (Table 1 ). Thus, control of NK activation may display similarities between host species, with some NK-evasins functioning across species barriers. This is consistent with reports of SARS-CoV2 being a 'generalist' virus that did not need to undergo extensive evolution to be capable of human-to-human transmission ${ }^{43}$.

\begin{tabular}{|llll|}
\hline Gene & Homo sapiens ID & $\begin{array}{l}\text { Rhinolophus } \\
\text { ferrumequinum ID }\end{array}$ & \% Identity \\
\hline B7-H6 & XP_011518377 & XP_032975102 & 52.19 \\
\hline Nectin 1 & NP_002846 & XP_032976402 & 96.12 \\
\hline MICA & NP_000238 & XP_032955521 & 43.41 \\
\hline ULBP2 & NP_079493 & XP_032959100 (ULBP1) & 42.26 \\
& & XP_032958641 (ULBP3) & 44.86 \\
\hline
\end{tabular}

Table 1. Homology of NK ligands from humans with those of the horseshoe bat

\section{NK-cell evasion is overcome by antibody-dependent activation}

In addition to their role in innate immunity through interactions with activating and inhibitory NK receptors, NK cells play additional roles in adaptive immunity via ADNKA/ADCC, in which CD16 interacts with the Fc portion of antibodies bound to targets on the surface of infected cells. To analyse this aspect of NK function, PBMC were incubated with infected cells in the presence of sera from individuals that were seronegative or seropositive for SARS-CoV2. Degranulation (Fig 4A), as well as production of IFN $\gamma$ (Fig 4B) and TNFo (Fig 4C), were all significantly increased in the presence of serum containing anti-SARS-CoV2 antibodies, across a range of different NK donors (Fig 4D-F). Viral immune evasion can therefore be overcome 
through antibody dependent mechanisms, suggesting that in addition to neutralising virus, SARS-CoV2 antibodies may aid virus clearance through ADCC.

The magnitude of the neutralising antibody response to SARS-CoV2 correlates with severity of disease; individuals with more severe disease generate higher levels of neutralising antibody ${ }^{44-46}$. Although memory B-cell responses persist, levels of circulating antibody can decrease over time following resolution of disease, such that in some mildly infected people they become undetectable after a few months ${ }^{44-46}$. To determine whether the same was true of ADCC responses, we assessed ADNKA in a cohort of donors with known disease status, for whom longitudinal sera were available (Fig 4G). ADNKA responses were generally of greater magnitude in individuals with more severe disease, with responses remaining detectable during the 150-200 day follow-up. Amongst those with milder disease, strong initial responses were maintained over time, while weaker responses decreased, becoming virtually undetectable by around 200 days. There was a significant correlation between the ability of antibodies to neutralise SARS-CoV2, and to activate ADNKA in response to SARS-CoV2 (Fig $4 \mathrm{H})$, with a $\mathrm{R}^{2}$ value of 0.7 . Thus, although the antibodies mediating these activities may be induced in a similar manner, the antibodies mediating the two responses may not be identical.

\section{Multiple SARS-CoV2 ORFs mediate ADCC}

There has been a major focus on spike as an activator of $A D C C^{8-12,14-18}$. It is certainly a potential target during infection as both our PMP and flow cytometric analysis detected substantial levels of spike protein on the infected cell surface (Fig 5A). However, our studies with other viruses have demonstrated that although viral proteins involved in virion binding and entry are found on the cell surface, and can be bound by antibody, they are not necessarily the strongest mediators of ADCC activity ${ }^{26}$. PMP also detected Membrane (M), Nucleocapsid (N) and ORF3a on the cell surface, while bioinformatic analysis of the entire viral genome revealed a further five viral proteins that contained potential transmembrane domains or signal peptides. We therefore screened cells transfected with each of these genes for their ability to activate NK cells in an antibody dependent manner. We used sera from patients with severe covid19 disease, that had previously generated the strongest ADNKA signals against infected cells (Fig 5B). As expected, spike primed a substantial ADNKA response. However, in 
support of our PMP data, expression of ORF3a, Membrane, and Nucleocapsid, were also capable of priming ADNKA in the presence of serum containing SARS-CoV2 antibodies.

Interestingly, nucleocapsid readily activated ADNKA despite the extremely low levels of this protein on the surface of transfected cells. We have previously observed the exquisite sensitivity of ADNKA, which can be activated in response to levels of virus protein that are difficult to detect by flow cytometry ${ }^{26}$. We have also shown that antigen levels can be very different in the context of virus infection, compared with transfection ${ }^{26}$. In support of this, nucleocapsid levels were much higher on the surface of infected cells (Fig 5C). Given how well even very low levels of nucleocapsid activated ADNKA, this suggested that multiple proteins other than spike could be major contributors to ADCC during SARS-CoV2 infection.

\section{Monoclonal anti-spike antibodies only weakly mediate ADNKA despite binding strongly to infected} cells

To determine the relative contribution of spike, and of different antigenic sites on Spike, to ADNKA during SARS-CoV2 infection, we tested a panel of 26 human anti-spike monoclonal antibodies, which had been isolated from naturally infected donors, and cloned as IgG1 constructs ${ }^{47}$. The panel included antibodies targeting the RBD, S1, NTD, and S2 domains, neutralising and non-neutralising, and those targeting multiple distinct epitopes (Table S3).

All 26 antibodies bound efficiently to infected cells, at levels comparable to polyclonal serum (Fig S3). However, only five of these antibodies were capable of triggering degranulation (Fig 6A), of which two triggered TNFa (Fig 6B), and one IFN $\gamma$ (Fig 6C). Importantly, these NK responses were at significantly lower levels than seen with polyclonal serum from a naturally infected donor. This suggested that, despite high levels of antibody binding, and a substantial ability to activate ADCC when expressed in isolation, spike may be a relatively poor ADCC target in the context of natural infection.

\section{Following natural infection, ADNKA responses are dominated by non-spike antibodies}

Although our data using monoclonal antibodies indicated that spike was a comparatively weak activator of ADNKA, the antibodies that mediate ADNKA may represent a minor proportion of the overall anti-spike response; we simply could not have captured those antibodies in our panel. To directly address this concern, we specifically depleted anti-spike 
antibodies from the serum of naturally infected individuals, and assessed changes in activity. ELISA analysis confirmed the effective depletion of spike-specific antibodies (Fig 7A), which was accompanied by a significant loss of neutralisation activity (Fig 7B), across multiple donors (Fig 7C). However, there was no loss of ADNKA activity from any of these samples following spike-antibody depletion (Fig 7D, E), consistent with the results of our monoclonal antibody data. We conclude that spike-specific antibodies dominate the neutralising antibody response, but play a minor roles in the ADCC response following natural infection.

\section{Spike-specific antibodies following vaccination are weak mediators of ADNKA}

Since non-spike antigens must prime ADCC following natural infection, we investigated individuals who have been vaccinated against spike, but not exposed to the virus - since they will only have spike-specific antibodies. As with serum from naturally infected individuals, ELISA demonstrated effective depletion of spike antibodies (Fig 7F), which was accompanied by a marked loss of virus neutralisation activity (Fig 7G, H). In functional NK assays, these sera were capable of mediating relatively weak ADNKA (Fig 7I, J) and, unlike sera from naturally infected individuals, ADNKA activity was abrogated following depletion of spike antibodies. When we compared responses following natural infection or vaccination, vaccine mediated ADNKA was significantly weaker than responses seen following natural infection, and was not boosted following the second dose of the vaccine (Fig 7K). This was in stark contrast with the neutralisation activity of those same serum samples, which were dramatically increased following the second dose, with activity comparable to patients with severe covid-19 disease (Fig 7L).

Thus, anti-spike antibodies can mediate ADNKA, but this activity appears weaker than responses seen during natural infection, which targets a broader repertoire of viral antigens. 


\section{Discussion}

The host innate immune system plays a critical role in both early SARS-CoV2 infection, and later COVID-19 disease. This is emphasised by the identification of multiple interferon antagonists encoded by SARS-CoV2, and the increased disease severity in patients with genetic and acquired interferon deficiencies ${ }^{3-6}$. To determine whether the virus evades other components of the host immune system we took a proteomic approach to systematically analyse how SARS-CoV2 infection manipulates the cell surface during the time course of SARSCoV2 infection. We find that SARS-CoV-2 remodelling of the plasma membrane leads to the downregulation of multiple cell surface immune ligands. This likely enables SARS-CoV2 to evade different effector arms of the host immune system. Downregulation of NK activating ligands inhibits NK cell activation early in infection, but this effect is countered later with the production of antibody and subsequent ADCC. Somewhat surprisingly, we find that spike antibody is not responsible for the robust ADNKA which develops after natural infection, and only weak ADNKA is generated following spike vaccination.

Genetic variation in both viruses and their hosts affects the ability of viruses to modulate NK responses, and influence pathogenesis ${ }^{1,48}$. SARS-CoV2 appears to have evolved to antagonise NK activation by targeting the expression levels of multiple activating NK ligands. All four of the NK ligands targeted by the virus are also found in bat species, thought to be the reservoir for most coronaviruses, implying an element of cross-species functionality to viral immuneevasins, which may be important for zoonotic transmission. Indeed, the $96 \%$ sequence identity between the nectin-1/CD111 bat and human genes is striking. Nectin-1 is a ligand for CD96/TACTILE ${ }^{49}$, which activates human NK cells ${ }^{50}$. SARS-CoV-2 also targets at least 3 additional NK activating ligands (B7-H6, ULBP2 and MICA). Of these, MICA is highly polymorphic (100+ alleles), with A549 cells expressing MICA*001/004 ${ }^{51}$, while the truncated MICA*008 is the most common allele worldwide. Further work will be needed to determine whether MICA genetic diversity affects the extremely variable outcome of SARS-CoV-2 infection.

In contrast to the effect on NK ligands, our data imply that the virus cannot actively antagonise adaptive cellular immunity. Our evidence for this includes the lack of significant MHC-I cell surface downregulation, the emergence of ADNKA with humoral immunity, and the inability 
of infected cells to bind human IgG from seronegative donors (i.e. the virus does not encode Fc receptors as ADNKA decoys). This is consistent with SARS-CoV2 acting as an acute 'hit and run' virus, which has replicated and transmitted before host adaptive immunity develops. It will be interesting to determine whether coronaviruses evade innate and adaptive immunity in their original bat host, and whether this contributes to their persistence in that species.

Animal models show that in both vaccination ${ }^{13}$, and monoclonal antibody administration ${ }^{19-22}$, Fc-mediated activation of cellular immunity plays an important protective role. However, inflammation dominates severe COVID-19 disease ${ }^{52,53}$. The high levels of TNF $\alpha$ and IFN $\gamma$ observed following ADNKA are likely to exacerbate disease under these circumstances. In support of this, TNF $\alpha$ and IFN $\gamma$ correlate with severe disease ${ }^{54}$, and critically ill patients have a higher proportion of afucosulated antibodies that promote a stronger ADCC response. Given that $A D C C$ can potentially contribute to both protection and immunopathology, it is important to understand what determines the magnitude and quality of the ADCC response. Viral entry glycoproteins are a common focus as mediators of ADCC. E.g. ADCC mediated by Env antibodies have been a major focus in $\mathrm{HIV}^{55}$, and gB-specific antibodies have been studied in $\mathrm{HCMV}^{56}$. Although antibodies targeting these antigens can clearly bind infected cells, using similar approaches to the present study we recently showed that non-structural accessory proteins were more potent ADCC targets during HCMV infection ${ }^{26}$. This conclusion is reinforced here, where the neutralising antibody response is dominated by spike, but spike antibodies are poor activators of ADNKA, with other viral antigens driving efficient ADNKA following natural infection. Nucleocapsid, ORF3a, and membrane are all present on the infected cell surface, and can promote ADNKA if appropriate antibodies are present. However, antibodies targeting ORF3a and membrane are only detected in a small subset of patients, while almost everyone infected with SARS-CoV2 develops antibodies targeting ${ }^{57}$. Nucleocapsid is therefore likely to be the major target for ADCC during natural SARS-CoV2 infection in most people. Influenza nucleoprotein is also detected at the plasma membrane ${ }^{58-}$ ${ }^{60}$, and antibodies targeting nucleoprotein can mediate ADCC ${ }^{61,62}$. Structural proteins involved in genome packaging may therefore represent a common target for ADCC across multiple virus families. 
The identification of the antigens that drive ADCC is important to our understanding of the mechanisms that underlie pathogenesis and disease. A skewing of the spike:nucleocapsid antibody ratio is associated with severe disease ${ }^{63-65}$, where ADCC-mediating nucleocapsid antibodies might be promoting inflammation. Both the breadth of the nucleocapsid antibody response, and the particular epitopes targeted, have also been correlated with disease severity ${ }^{57,66}$. These correlations now need to be followed up functionally, to determine how the epitopes bound by anti-nucleocapsid antibodies affect ADCC. It will also be important to determine the contribution of these ADCC-capable antibodies to inflammation and myeloid cell activation ${ }^{67-69}$, via antibody-dependent cellular phagocytosis (ADCP) and complement dependent cytolysis (CDC).

Although Fc-dependent mechanisms of cellular immune activation could contribute to immunopathology via multiple mechanisms in severe disease, all patients with mild/asymptomatic disease also developed strong and persistent ADNKA responses. Furthermore, lineage defining mutations in the viral variants of concern B.1.1.7, B.1.351, and P.1, all include non-synonymous mutations in nucleocapsid, which is one of the most variable genes amongst circulating strains ${ }^{70}$, and these nucleocapsid mutations can result in reduced antibody binding ${ }^{71}$. Virus variants may therefore evolve to evade ADCC responses as well as neutralising responses, supporting an important role for ADCC in protection. It is therefore significant that spike-vaccine generated antibody responses were poor ADNKA inducers. The addition of other viral proteins such as nucleocapsid to vaccines would engage a wider range of immune effector pathways. This approach might improve efficacy against both viral transmission and disease, resulting in vaccines that are more resistant to viral variants containing mutations that diminish antibody neutralisation ${ }^{72}$. 


\section{Acknowledgements}

This work was supported in part by the UK Coronavirus Immunology Consortium (CiC). RS was supported by the MRC (MR/S00971X/1), and Wellcome Trust (204870/Z/16/Z), PJL was funded by the Wellcome Trust through a Principal Research Fellowship [210688/Z/18/Z], the MRC (MR/V011561/1), the Addenbrooke's Charitable Trust and the NIHR Cambridge Biomedical Research Centre. KLD was supported by King's Together Rapid COVID-19 Call, Huo Family Foundation Award, and a Wellcome Trust Multi-User Equipment Grant 208354/Z/17/Z. CG was supported by the MRC-KCL Doctoral Training Partnership in Biomedical Sciences (MR/N013700/1). BM was supported by an NIHR Academic Clinical Fellowship in Combined Infection Training 


\section{Materials and methods}

\section{Cells and viruses}

A549 were transduced with lentiviruses expressing human ACE2, and TMPRSS2 (AAT cells), as previously described ${ }^{27}$. The England2 strain of SARS-CoV2 was obtained from Public Health England (PHE), grown on VeroE6 cells, and titrated by plaque assay on both VeroE6 and AAT, as previously described ${ }^{27}$. All cell lines were grown in DMEM containing 10\% FCS (Gibco), at $37^{\circ} \mathrm{C}$ and in $5 \% \mathrm{CO}_{2}$. For assays, cells were plated out the day before, then infected at a multiplicity of infection (MOI) of 5 , for 1 h on a rocking platform. The inoculum was removed, and fresh DMEM containing 2\% FCS was added, then cells were incubated until the assay.

\section{Plasma membrane profiling}

Cell surface proteins were labelled essentially as described ${ }^{73}$. Briefly, cells were incubated in a solution containing sodium periodate, aniline and aminooxy biotin to label predominantly sialic acid containing glycans at the cell surface. Cell lysates were then enriched for labelled proteins using streptavidin-agarose beads. After extensive washing trypsin was added to liberate peptides of the enriched proteins. The resulting peptide pools were dried prior to labelling with TMT reagents.

\section{TMT Labelling and clean-up}

Samples were resuspended in $21 \mu \mathrm{L} 100 \mathrm{mM}$ TEAB pH 8.5. After allowing to come to room temperature, 0.2mg TMT reagents (Thermo Fisher) were resuspended in $9 \mu \mathrm{L}$ anhydrous ACN which was added to the respective samples and incubated at room temperature for $1 \mathrm{~h}$. A $3 \mu \mathrm{L}$ aliquot of each sample was taken and pooled to check TMT labelling efficiency and equality of loading by LC-MS. After checking each sample was at least $98 \%$ TMT labelled total reporter ion intensities were used to normalise the pooling of the remaining samples such that the final pool was as close to a 1:1 ratio of total peptide content between samples as possible. This final pool was then dried in a vacuum centrifuge to evaporate the majority of ACN form labelling. The sample was acidified to a final 0.1\% Trifluoracetic Acid (TFA) ( 200 $\mu$ L volume) and FA was added until the SDC visibly precipitated. 4 volumes of ethyl acetate were then added and the sample vortexed vigorously for 10 s. Sample was then centrifuged at $15^{\prime} 000 \mathrm{~g}$ for 5 mins at RT to effect phase separation. A gel loading pipette tip was used to withdraw the lower (aqueous) phase to a fresh low adhesion microfuge tube. The sample was then partially 
dried in a vacuum centrifuge and brought up to a final volume of $1 \mathrm{~mL}$ with $0.1 \%$ TFA. FA was added until the $\mathrm{pH}$ was $<2$, confirmed by spotting onto $\mathrm{pH}$ paper. The sample was then cleaned up by SPE using a 50mg tC18 SepPak cartridge (Waters). The cartridge was wetted with $1 \mathrm{~mL} 100 \%$ Methanol followed by $1 \mathrm{~mL}$ ACN, equilibrated with $1 \mathrm{~mL} 0.1 \%$ TFA and the sample loaded slowly. The sample was passed twice over the cartridge. The cartridge was washed $3 x$ with $1 \mathrm{~mL} 0.1 \%$ TFA before eluting sequentially with $250 \mu \mathrm{L} 40 \% \mathrm{ACN}, 70 \% \mathrm{ACN}$ and $80 \% \mathrm{ACN}$ and dried in a vacuum centrifuge.

\section{Basic $\mathrm{pH}$ reversed phase fractionation}

TMT labelled samples were resuspended in 40 $\mu \mathrm{L} 200 \mathrm{mM}$ Ammonium formate $\mathrm{pH} 10$ and transferred to a glass HPLC vial. BpH-RP fractionation was conducted on an Ultimate 3000 UHPLC system (Thermo Scientific) equipped with a $2.1 \mathrm{~mm} \times 15 \mathrm{~cm}, 1.7 \mu$ Kinetex EVO column (Phenomenex). Solvent A was $3 \%$ ACN, Solvent B was $100 \% A C N$, solvent $C$ was $200 \mathrm{mM}$ ammonium formate ( $\mathrm{pH} 10)$. Throughout the analysis solvent $\mathrm{C}$ was kept at a constant $10 \%$. The flow rate was $500 \mu \mathrm{L} / \mathrm{min}$ and UV was monitored at $280 \mathrm{~nm}$. Samples were loaded in $90 \%$ A for 10 min before a gradient elution of 0-10\% B over 10 min (curve 3), 10-34\% B over 21 min (curve 5), 34-50\% B over 5 mins (curve 5) followed by a 10 min wash with $90 \%$ B. $15 \mathrm{~s}$ $(100 \mu \mathrm{L})$ fractions were collected throughout the run. Fractions containing peptide (as determined by $\mathrm{A} 280$ ) were recombined across the gradient to preserve orthogonality with on-line low pH RP separation. For example, fractions 1, 25, 49, 73, 97 are combined and dried in a vacuum centrifuge and stored at $-20^{\circ} \mathrm{C}$ until LC-MS analysis. 24 Fractions were generated in this manner.

\section{Mass Spectrometry}

Samples were analysed on an Orbitrap Fusion instrument on-line with an Ultimate 3000 RSLC nano UHPLC system (Thermo Fisher). Samples were resuspended in 10 $\mu \mathrm{L}$ 5\% DMSO/1\% TFA and all sample was injected. Trapping solvent was $0.1 \%$ TFA, analytical solvent $A$ was $0.1 \%$ FA, solvent B was ACN with 0.1\% FA. Samples were loaded onto a trapping column $(300 \mu \mathrm{m} \times 5 \mathrm{~mm}$ PepMap cartridge trap (Thermo Fisher)) at $10 \mu \mathrm{L} / \mathrm{min}$ for 5 minutes at 60 degrees. Samples were then separated on a $75 \mathrm{~cm}$ x $75 \mu \mathrm{m}$ i.d. $2 \mu \mathrm{m}$ particle size PepMap C18 column (Thermo Fisher) at 55 degrees. The gradient was 3-10\% B over 10mins, 10-35\% B over 155 minutes, 35$45 \%$ B over 9 minutes followed by a wash at $95 \%$ B for 5 minutes and requilibration at 3\% B. 
Eluted peptides were introduced by electrospray to the MS by applying $2.1 \mathrm{kV}$ to a stainlesssteel emitter $(5 \mathrm{~cm} \times 30 \mu \mathrm{m}$ (PepSep)). During the gradient elution, mass spectra were acquired with the parameters detailed in Fig S4 using Tune v3.3 and Xcalibur v4.3 (Thermo Fisher).

\section{Data Processing}

Data were processed with PeaksX+, v10.5 (Bioinformatic Solutions). Processing parameters are shown in detail in Supplementary Fig S5. Briefly, .raw files were searched iteratively in three rounds, with unmatched de novo spectra (at 1\% PSM FDR) from the previous search used as the input for the next. The three iterations were as follows 1) Swissprot Human $(27 / 03 / 2020)+$ common contaminants 2$)$ The same databases as search 1 but permitting semi-specific cleavage 3) trEMBL Human (27/03/2020), with specific cleavage rules. Proteins were then quantified using the parameters outlined in Fig S5. Identified proteins and their abundances were output to .csv format and further subjected to statistical analysis. The mass spectrometry proteomics data have been deposited to the ProteomeXchange Consortium via the PRIDE partner repository ${ }^{74}$ with the dataset identifier PXD025000 and 10.6019/PXD025000. Table S1 contains the analysed data.

\section{Statistical analysis and K-means clustering.}

Prior to statistical analysis, proteins were filtered for those quantified across all TMT reporter channels and with more than 1 unique. Only proteins with a plasma membrane related GO:CC annotation as previously described ${ }^{28}$ were then carried forward for statistical analysis. All SARS-CoV-2 proteins quantified with more than one unique peptide were also included. Statistical tests were performed with the aov and p.adjust functions in the $\mathrm{R}$ base stats package (version 4.0.3) to calculate Benjamini-Hochberg corrected p-values for changes in protein abundance across the measured time-points.

Proteins with a $p$-value $<0.05$ and a maximum fold-change across the time-course of $>1.5$ fold were selected for k-means clustering. Mean intensities of each time-point were scaled using the scale function in base $\mathrm{R}$ and utilised to cluster proteins into 5 groups using the kmeans function in $\mathrm{R}$ base stats package.

\section{Functional Annotation Clustering}


Assessment of enriched gene annotation terms in temporal cluster one was carried out using the Functional Annotation Clustering tool at DAVID (david.ncifcrf.gov) v6.875, using the default clustering settings for medium stringency and the following libraries: Uniprot UP_Keyword, GOTERM:MF_ALL, BIOCARTA,, KEGG_PATHWAY and REACTOME_PATHWAY. Output clusters were curated with representative names based on the enriched terms within. The background for enrichment was a list of proteins detected in the PMP dataset with more than one peptides and a plasma membrane associated GO:CC term. Functional annotation clusters with an enrichment score of $>1.5$ are shown, and any clusters in which no individual annotation term had a Benjamini-Hochberg correct $p$-value of $<0.05$ were excluded.

\section{Flow cytometry}

Cells were dissociated using TrypLE, then stained with primary antibody for 30 min at $4^{\circ} \mathrm{C}$. Following washing, they were incubated with secondary antibody, again for 30 min at $4^{\circ} \mathrm{C}$. Cells were washed, fixed in $4 \%$ paraformaldehyde, and data collected on an Attune cytometer (Thermofisher). Antibodies used were against HLA-ABC (W632; AbD Serotec), NCR3LG1/B7H6 (MAB7144, Biotechne R\&D Systems), Nectin 1 (R1.302; Biolegend), MICA (AMO1-100; BAMOMAB), MICB (BMO2-100; BAMOMAB), ULBP2 (BUMO1; BAMOMAB), Spike (1A9; Insight), Nucleocapsid (1C7; Stratech), anti-mouse IgG AF647 (Thermofisher).

\section{Plasmids and transfections}

Lentivirus plasmids encoding each SARS-CoV2 ORF individually, with a C-terminal twin-strep tag, were obtained from Addgene ${ }^{76}$. Plasmids were midiprepped (Nucleobond Xtra Midi; Machery-Nagel), and transfected into 293T cells using GeneJuice (Merck) according to manufacturers' instructions.

\section{NK activation assays}

PBMCs from healthy donors were thawed from liquid N2 storage, rested overnight in RPMI supplemented with $10 \%$ FCS, and L-glutamine $(2 \mathrm{mM})$ in the absence or presence of IFN- $\alpha$ $(1,000 \mathrm{U} / \mathrm{ml})$. Target cells were harvested using TrypLE Express (Gibco), preincubated for 30 min with the relevant antibody or serum preparations, then mixed with PBMCs at an effector:target $(E: T)$ ratio of 10:1 in the presence of GolgiStop $(0.7 \mu \mathrm{l} / \mathrm{ml}$, BD Biosciences), Brefeldin-A (1:1000, Biolegend) and anti-CD107a-FITC (clone H4A3, BioLegend). Cells were 
incubated for $5 \mathrm{~h}$, washed in cold PBS, and stained with live/dead Fixable Aqua (Thermo Fisher Scientific), anti-CD3-PECy7 (clone UCHT1, BioLegend) or anti-CD3-BV711 (Clone SK7, Biolegend), anti-CD56-BV605 (clone 5.1H11, BioLegend), anti-CD57-APC (clone HNK-1, BioLegend) or anti-CD57-PECy7 (clone HNK-1, biolegend), and anti-NKG2C-PE (clone 134591, R\&D Systems). In some experiments, cells were also fixed/permeabilized using Cytofix/Cytoperm (BD Biosciences) and stained with anti-TNF $\alpha-B V 421$ (clone MAb11, BioLegend) and anti-IFNY-APC (clone 4S.B3, BioLegend). Data were acquired using an AttuneNxT (Thermo Fisher) and analyzed with Attune NxT software or FlowJo software version 10 (Tree Star). To ensure inter-assay variation did not affect results, a donor serum demonstrating moderate ADCC activity against SARS-CoV2 was included as a positive standard in every assay, while a serum collected before 2020 was included as a negative control in every assay. Where sera were tested at a range of dilutions, the area under the curve (AUC) was calculated using Graphpad Prism 9. This value was then normalised to the AUC for the standard serum in that particular assay.

\section{Serums}

Serums were collected from healthy donors vaccinated with the BNT162b2 vaccine, a minimum of 3 weeks after the first dose, or 1 week after the second. A pre-vaccine sample was taken from every donor, and an ELISA for SARS-CoV2 RBD performed as previously described $^{77}$, to determine pre-exposure to live SARS-CoV2. Longitudinal serums from naturally infected individuals have been described previously, clinical characteristics are in Table S2 ${ }^{44}$. Those labelled 'mild' had severity scores of $0,1,2$ or 3, and 'severe' was 4 or 5.

\section{Virus neutralisation assays}

600PFU of SARS-CoV2 was incubated with appropriate dilutions of serum, for $1 \mathrm{~h}$, at $37^{\circ} \mathrm{C}$. The mixes were then added to pre-plated VeroE6 cells for $48 \mathrm{~h}$. After this time, monolayers were fixed with 4\% PFA, permeabilised for 15 min with $0.5 \%$ NP-40, then blocked for $1 \mathrm{~h}$ in PBS containing $0.1 \%$ Tween (PBST) and 3\% non-fat milk. Primary antibody (anti-nucleocapsid 1C7, Stratech, 1:500 dilution) was added in PBST containing $1 \%$ non-fast milk and incubated for $1 \mathrm{~h}$ at room temperature. After washing in PBST, secondary antibody (anti-mouse IgG-HRP, Pierce, 1:3,000 dilution) was added in PBST containing $1 \%$ non-fat milk and incubated for $1 \mathrm{~h}$. Monolayers were washed again, developed using Sigmafast OPD according to manufacturers' 
instructions, and read on a Clariostar Omega plate reader. Wells containing no virus, virus but no antibody, and a standardised serum displaying moderate activity were included as controls in every experiment. NT50 were calculated in Graphpad Prism 9.

\section{Antibody depletions}

Anti-spike antibody was depleted from sera using magnetic bead conjugated spike trimer protein (Acrobiosystems). Beads were resuspended in PBS+0.05\% BSA, then 50 $\mu$ l serum was mixed with $150 \mu \mathrm{l}$ beads. Mixtures were incubated on a rotating mixer at $4^{\circ} \mathrm{C}$ overnight. Serum diluted in buffer alone was used as a control. Magnetic beads were then removed using a 3D printed magnetic stand. All values given in assays are corrected for this initial 4-fold dilution. Levels of anti-spike antibody were measured using a spike trimer ELISA (Acrobiosystems).

\section{Ethics}

PBMC were extracted from apheresis cones obtained from the Welsh Blood Service (WBS) via an ad-hoc agreement or from blood samples from healthy volunteers and stored in liquid $\mathrm{N}_{2}$ until use. Use of healthy volunteer PBMC for this project, including those from WBS, was ethically approved by the Cardiff University School of Medicine Research Ethics Committee (SMREC) nos. 20/55 and 20/101. Recruitment of healthy volunteers after vaccination was covered by the Cardiff University School of Medicine Research Ethics Committee under reference no. 18/04. 


\section{$\underline{\text { References }}$}

Patel, M. et al. HCMV-Encoded NK Modulators: Lessons From in vitro and in vivo Genetic Variation. Frontiers in immunology 9, 2214, (2018).

Berry, R., Watson, G. M., Jonjic, S., Degli-Esposti, M. A. \& Rossjohn, J. Modulation of innate and adaptive immunity by cytomegaloviruses. Nat Rev Immunol 20, 113-127, (2020).

Sa Ribero, M., Jouvenet, N., Dreux, M. \& Nisole, S. Interplay between SARS-CoV-2 and the type I interferon response. PLoS Pathog 16, e1008737, (2020).

Miorin, L. et al. SARS-CoV-2 Orf6 hijacks Nup98 to block STAT nuclear import and antagonize interferon signaling. Proc Natl Acad Sci U S A 117, 28344-28354, (2020).

Schroeder, S. et al. Interferon antagonism by SARS-CoV-2: a functional study using reverse genetics. The Lancet Microbe, (2021).

Lei, X. et al. Activation and evasion of type I interferon responses by SARS-CoV-2. Nature communications 11, 3810, (2020).

Kumar, S. Natural killer cell cytotoxicity and its regulation by inhibitory receptors. Immunology 154, 383-393, (2018).

Dufloo, J. et al. Asymptomatic and symptomatic SARS-CoV-2 infections elicit polyfunctional antibodies. medRxiv, 2020.2011.2012.20230508, (2020).

Herman, J. D. et al. Functional Antibodies in COVID-19 Convalescent Plasma. medRxiv, 2021.2003.2008.21253157, (2021).

Lee, W. S. et al. Decay of Fc-dependent antibody functions after mild to moderate COVID-19. medRxiv, 2020.2012.2013.20248143, (2020).

1 Anand, S. P. et al. Longitudinal analysis of humoral immunity against SARS-CoV-2 Spike in convalescent individuals up to 8 months post-symptom onset. bioRxiv, 2021.2001.2025.428097, (2021).

Zohar, T. et al. Compromised Humoral Functional Evolution Tracks with SARS-CoV-2 Mortality. Cell 183, 1508-1519 e1512, (2020).

Gorman, M. J. et al. Collaboration between the Fab and Fc contribute to maximal protection against SARS-CoV-2 in nonhuman primates following NVX-CoV2373 subunit vaccine with Matrix-M vaccination. bioRxiv, 2021.2002.2005.429759, (2021).

Barrett, J. R. et al. Phase 1/2 trial of SARS-CoV-2 vaccine ChAdOx1 nCoV-19 with a booster dose induces multifunctional antibody responses. Nat Med 27, 279-288, (2021).

Tauzin, A. et al. A single BNT162b2 mRNA dose elicits antibodies with Fc-mediated effector functions and boost pre-existing humoral and $\mathrm{T}$ cell responses. bioRxiv, 2021.2003.2018.435972, (2021).

Tortorici, M. A. et al. Ultrapotent human antibodies protect against SARS-CoV-2 challenge via multiple mechanisms. Science 370, 950-957, (2020).

McCallum, M. et al. N-terminal domain antigenic mapping reveals a site of vulnerability for SARS-CoV-2. bioRxiv, 2021.2001.2014.426475, (2021).

8 Cathcart, A. L. et al. The dual function monoclonal antibodies VIR-7831 and VIR-7832 demonstrate potent in vitro and in vivo activity against SARS-CoV-2. bioRxiv, 2021.2003.2009.434607, (2021).

9 Chan, C. E. Z. et al. The Fc-mediated effector functions of a potent SARS-CoV-2 neutralizing antibody, SC31, isolated from an early convalescent COVID-19 patient, are essential for the optimal therapeutic efficacy of the antibody. bioRxiv, 2020.2010.2026.355107, (2020).

Schafer, A. et al. Antibody potency, effector function, and combinations in protection and therapy for SARS-CoV-2 infection in vivo. J Exp Med 218, (2021).

Winkler, E. S. et al. Human neutralizing antibodies against SARS-CoV-2 require intact Fc effector functions and monocytes for optimal therapeutic protection. bioRxiv, 2020.2012.2028.424554, (2020). 
22 Suryadevara, N. et al. Neutralizing and protective human monoclonal antibodies recognizing the N-terminal domain of the SARS-CoV-2 spike protein. bioRxiv, 2021.2001.2019.427324, (2021).

23 Hammer, Q. \& Romagnani, C. OMIP-039: Detection and analysis of human adaptive NKG2C(+) natural killer cells. Cytometry A 91, 997-1000, (2017).

24 Maucourant, C. et al. Natural killer cell immunotypes related to COVID-19 disease severity. Sci Immunol 5, eabd6832, (2020).

25 Vietzen, H. et al. Deletion of the NKG2C receptor encoding KLRC2 gene and HLA-E variants are risk factors for severe COVID-19. Genet Med, (2021).

26 Vlahava, V. M. et al. Monoclonal antibodies targeting nonstructural viral antigens can activate ADCC against human cytomegalovirus. J Clin Invest 131, (2021).

27 Rihn, S. J. et al. A plasmid DNA-launched SARS-CoV-2 reverse genetics system and coronavirus toolkit for COVID-19 research. PLoS Biol 19, e3001091, (2021).

28 Weekes, M. P. et al. Comparative analysis of techniques to purify plasma membrane proteins. J Biomol Tech 21, 108-115, (2010).

29 Matheson, N. J. et al. Cell Surface Proteomic Map of HIV Infection Reveals Antagonism of Amino Acid Metabolism by Vpu and Nef. Cell host \& microbe 18, 409-423, (2015).

30 Fielding, C. A. et al. Control of immune ligands by members of a cytomegalovirus gene expansion suppresses natural killer cell activation. Elife 6, (2017).

31 Hsu, J. L. et al. Plasma membrane profiling defines an expanded class of cell surface proteins selectively targeted for degradation by HCMV US2 in cooperation with UL141. PLoS Pathog 11, e1004811, (2015).

32 Weekes, M. P. et al. Quantitative temporal viromics: an approach to investigate hostpathogen interaction. Cell 157, 1460-1472, (2014).

33 Weekes, M. P. et al. Latency-associated degradation of the MRP1 drug transporter during latent human cytomegalovirus infection. Science 340, 199-202, (2013).

34 Soh, T. K. et al. Temporal Proteomic Analysis of Herpes Simplex Virus 1 Infection Reveals CellSurface Remodeling via pUL56-Mediated GOPC Degradation. Cell reports 33, 108235, (2020). Patra, T. et al. SARS-CoV-2 spike protein promotes IL-6 trans-signaling by activation of angiotensin II receptor signaling in epithelial cells. PLoS Pathog 16, e1009128, (2020). Greenwood, E. J. et al. Temporal proteomic analysis of HIV infection reveals remodelling of the host phosphoproteome by lentiviral Vif variants. eLife 5, (2016).

37 Weekes, M. P. et al. Quantitative temporal viromics: an approach to investigate hostpathogen interaction. Cell 157, 1460-1472, (2014).

38 Sarrazin, S., Lamanna, W. C. \& Esko, J. D. Heparan sulfate proteoglycans. Cold Spring Harb Perspect Biol 3, (2011).

39 Cantuti-Castelvetri, L. et al. Neuropilin-1 facilitates SARS-CoV-2 cell entry and infectivity. Science 370, 856-860, (2020).

40 Daly, J. L. et al. Neuropilin-1 is a host factor for SARS-CoV-2 infection. Science 370, 861-865, (2020).

41 Clausen, T. M. et al. SARS-CoV-2 Infection Depends on Cellular Heparan Sulfate and ACE2. Cell 183, 1043-1057 e1015, (2020).

42 Landi, A., lannucci, V., Nuffel, A. V., Meuwissen, P. \& Verhasselt, B. One protein to rule them all: modulation of cell surface receptors and molecules by HIV Nef. Curr HIV Res 9, 496-504, (2011).

43 MacLean, O. A. et al. Natural selection in the evolution of SARS-CoV-2 in bats created a generalist virus and highly capable human pathogen. PLoS Biol 19, e3001115, (2021).

44 Seow, J. et al. Longitudinal observation and decline of neutralizing antibody responses in the three months following SARS-CoV-2 infection in humans. Nat Microbiol 5, 1598-1607, (2020).

45 Dan, J. M. et al. Immunological memory to SARS-CoV-2 assessed for up to 8 months after infection. Science 371, eabf4063, (2021). 
Wajnberg, A. et al. SARS-CoV-2 infection induces robust, neutralizing antibody responses that are stable for at least three months. medRxiv, 2020.2007.2014.20151126, (2020).

47 Graham, C. et al. Impact of the B.1.1.7 variant on neutralizing monoclonal antibodies recognizing diverse epitopes on SARS-CoV-2 Spike. bioRxiv, 2021.2002.2003.429355, (2021). Vietzen, H. et al. Extent of Cytomegalovirus Replication in the Human Host Depends on Variations of the HLA-E/UL40 Axis. MBio 12, (2021).

49 Seth, S. et al. The murine pan T cell marker CD96 is an adhesion receptor for CD155 and nectin1. Biochemical and Biophysical Research Communications 364, 959-965, (2007).

50 Holmes, V. M. et al. Interaction between nectin-1 and the human natural killer cell receptor CD96. PLoS One 14, e0212443, (2019).

51 Seidel, E. et al. Dynamic Co-evolution of Host and Pathogen: HCMV Downregulates the Prevalent Allele MICA*008 to Escape Elimination by NK Cells. Cell reports 10, 968-982, (2015).

52 Horby, P. W. et al. Tocilizumab in patients admitted to hospital with COVID-19 (RECOVERY): preliminary results of a randomised, controlled, open-label, platform trial. medRxiv, 2021.2002.2011.21249258, (2021).

53 Group, R. C. et al. Dexamethasone in Hospitalized Patients with Covid-19. N Engl J Med 384, 693-704, (2021).

54 Karki, R. et al. Synergism of TNF-alpha and IFN-gamma Triggers Inflammatory Cell Death, Tissue Damage, and Mortality in SARS-CoV-2 Infection and Cytokine Shock Syndromes. Cell 184, 149-168 e117, (2021).

55 Forthal, D. N. \& Finzi, A. Antibody-dependent cellular cytotoxicity in HIV infection. AIDS 32, 2439-2451, (2018).

56 Nelson, C. S. et al. HCMV glycoprotein B subunit vaccine efficacy mediated by nonneutralizing antibody effector functions. Proc Natl Acad Sci U S A 115, 6267-6272, (2018).

57 Shrock, E. et al. Viral epitope profiling of COVID-19 patients reveals cross-reactivity and correlates of severity. Science 370, (2020).

58 Yewdell, J. W., Frank, E. \& Gerhard, W. Expression of influenza A virus internal antigens on the surface of infected P815 cells. J Immunol 126, 1814-1819, (1981).

59 Fujimoto, Y. et al. Cross-protective potential of anti-nucleoprotein human monoclonal antibodies against lethal influenza A virus infection. J Gen Virol 97, 2104-2116, (2016).

60 Virelizier, J. L., Allison, A. C., Oxford, J. S. \& Schild, G. C. Early presence of ribonucleoprotein antigen on surface of influenza virus-infected cells. Nature 266, 52-54, (1977).

61 Vanderven, H. A. et al. What Lies Beneath: Antibody Dependent Natural Killer Cell Activation by Antibodies to Internal Influenza Virus Proteins. EBioMedicine 8, 277-290, (2016).

62 Jegaskanda, S. et al. Induction of H7N9-Cross-Reactive Antibody-Dependent Cellular Cytotoxicity Antibodies by Human Seasonal Influenza A Viruses that are Directed Toward the Nucleoprotein. The Journal of infectious diseases 215, 818-823, (2017).

63 Roltgen, K. et al. Defining the features and duration of antibody responses to SARS-CoV-2 infection associated with disease severity and outcome. Sci Immunol 5, eabe0240, (2020).

64 de Campos Mata, L. et al. SARS-CoV-2-Specific Antibody Profiles Distinguish Patients with Moderate from Severe COVID-19. medRxiv, 2020.2012.2018.20248461, (2020).

65 Atyeo, C. et al. Distinct Early Serological Signatures Track with SARS-CoV-2 Survival. Immunity 53, 524-532 e524, (2020).

66 Voss, C. et al. Epitope-resolved serology test differentiates the clinical outcome of COVID-19 and identifies defects in antibody response in SARS-CoV-2 variants. medRxiv, 2021.2003.2016.21253716, (2021).

67 Schulte-Schrepping, J. et al. Severe COVID-19 Is Marked by a Dysregulated Myeloid Cell Compartment. Cell 182, 1419-1440 e1423, (2020).

68 Szabo, P. A. et al. Longitudinal profiling of respiratory and systemic immune responses reveals myeloid cell-driven lung inflammation in severe COVID-19. Immunity, (2021). 
69 Merad, M. \& Martin, J. C. Pathological inflammation in patients with COVID-19: a key role for monocytes and macrophages. Nat Rev Immunol 20, 355-362, (2020).

70 Moore, C. et al. Localised community circulation of SARS-CoV-2 viruses with an increased accumulation of single nucleotide polymorphisms that adversely affect the sensitivity of realtime reverse transcription assays targeting Nucleocapsid protein. medRxiv, 2021.2003.2022.21254006, (2021).

71 Del Vecchio, C. et al. Emergence of $\mathrm{N}$ antigen SARS-CoV-2 genetic variants escaping detection of antigenic tests. medRxiv, 2021.2003.2025.21253802, (2021).

72 Madhi, S. A. et al. Efficacy of the ChAdOx1 nCoV-19 Covid-19 Vaccine against the B.1.351 Variant. N Engl J Med, (2021).

73 Weekes, M. P. et al. Proteomic plasma membrane profiling reveals an essential role for gp96 in the cell surface expression of LDLR family members, including the LDL receptor and LRP6. Journal of proteome research 11, 1475-1484, (2012).

74 Perez-Riverol, Y. et al. The PRIDE database and related tools and resources in 2019: improving support for quantification data. Nucleic Acids Res 47, D442-D450, (2019).

75 Huang da, W., Sherman, B. T. \& Lempicki, R. A. Systematic and integrative analysis of large gene lists using DAVID bioinformatics resources. Nat Protoc 4, 44-57, (2009).

76 Gordon, D. E. et al. A SARS-CoV-2 protein interaction map reveals targets for drug repurposing. Nature 583, 459-468, (2020).

77 Amanat, F. et al. A serological assay to detect SARS-CoV-2 seroconversion in humans. Nature Medicine 26, 1033-+, (2020). 


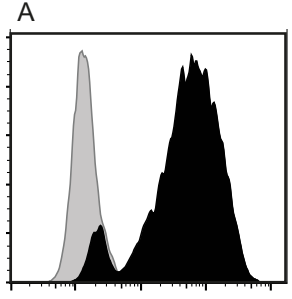

Spike

$\square$ Mock $\square$ Infected

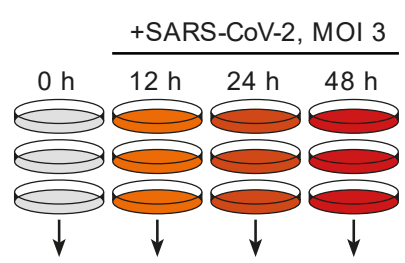

Plasma membrane enrichment anछMT-based quatification by $\mathrm{LC} / \mathrm{MS}^{3}$

2227 PM proteins quantified with >1 peptide $\downarrow$

ANOVA and K-means clustering

$$
\downarrow
$$

914 proteins changing significantly and by at least 1.5 -fold over the time-course, clustered into 5 groups

D
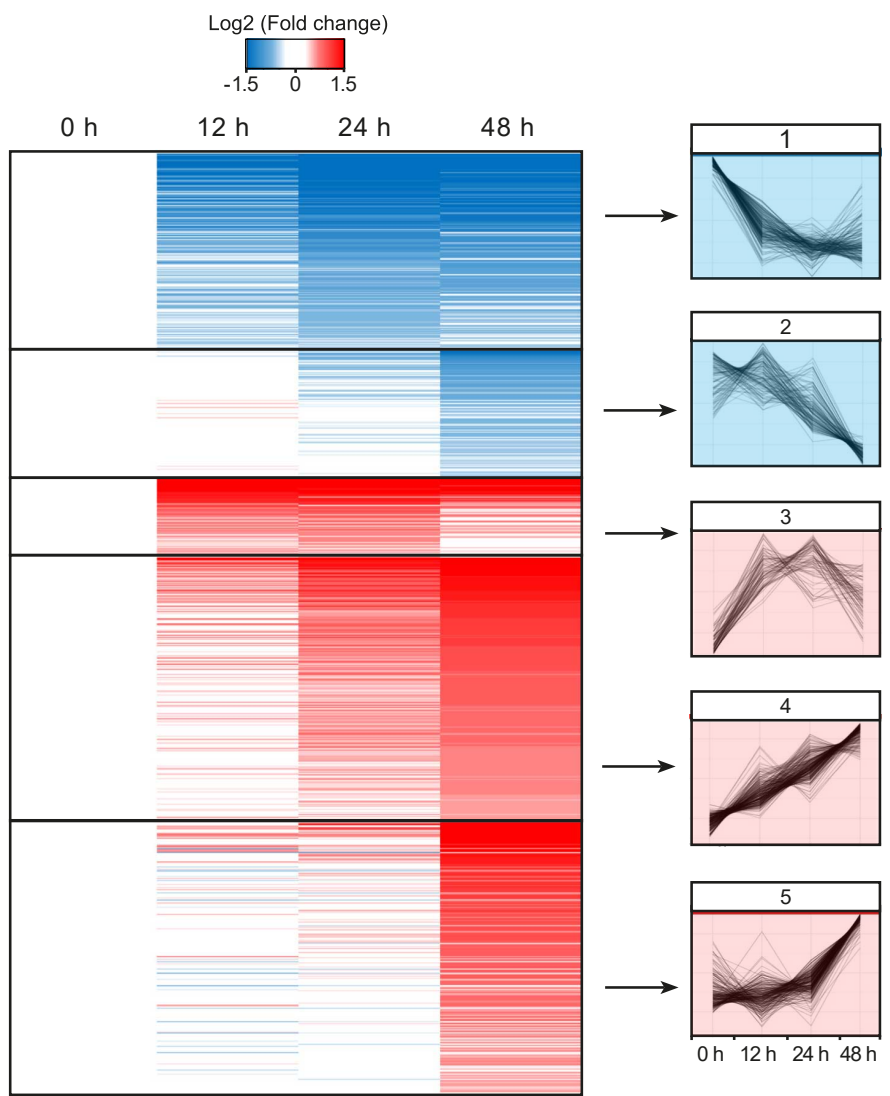

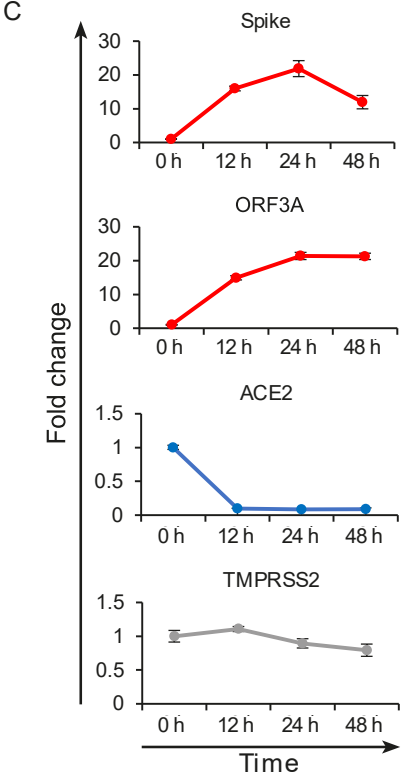

Fig 1. SARS-CoV-2 remodels the Plasma Membrane Proteome. (A) AAT cells were infected with SARSCoV2 in biological triplicate. 24h later they were detached with trypsin, fixed and permeabilised, stained for Spike protein, and analysed by flow cytometry. (B) Schematic of plasma membrane profiling and analysis pathway. C. Examples of temporal profiles of viral and cellular genes, fold change is compared to $0 \mathrm{~h}$ timepoint. Data points show mean \pm SD. D. Left - heat map of the 914 significantly changing proteins clustered by $\mathrm{k}$-mean, colour indicates log2 fold change compared to $0 \mathrm{~h}$, right, $\mathrm{Z}$ score normalised temporal profiles of proteins within each cluster. 


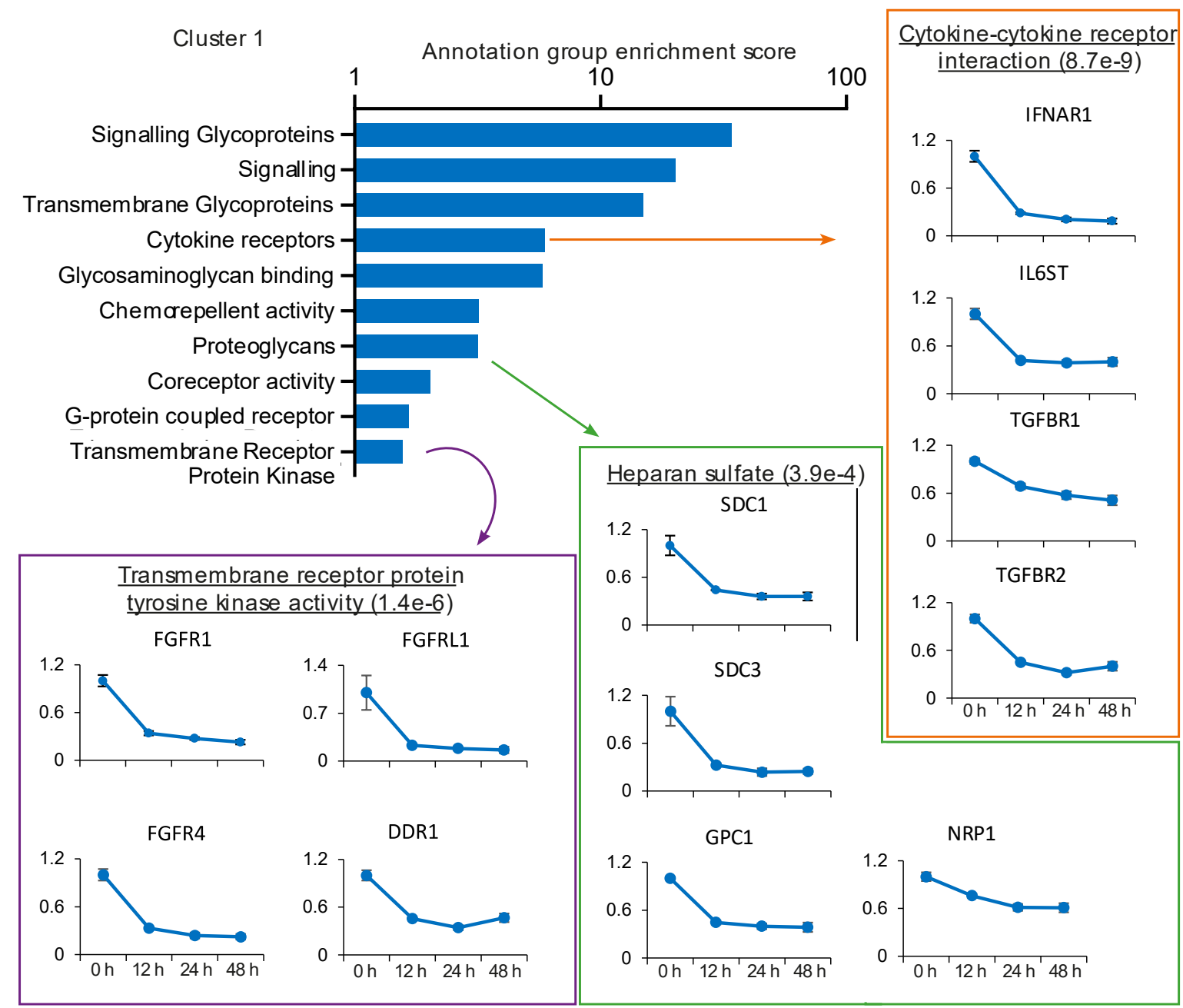

Figure 2. SARS-CoV2 manipulates multiple classes of plasma membrane proteins. (A) Enriched gene ontology and pathway annotations of proteins downregulated in temporal cluster 1 were condensed to groups of related terms, bar chart shows the enrichment score of these groups. Examples of annotation terms falling within each group are shown underlined with Bejamani-Hochberg adjusted P-values in parentheses, alongside four examples of proteins for each annotation. 


\section{A}
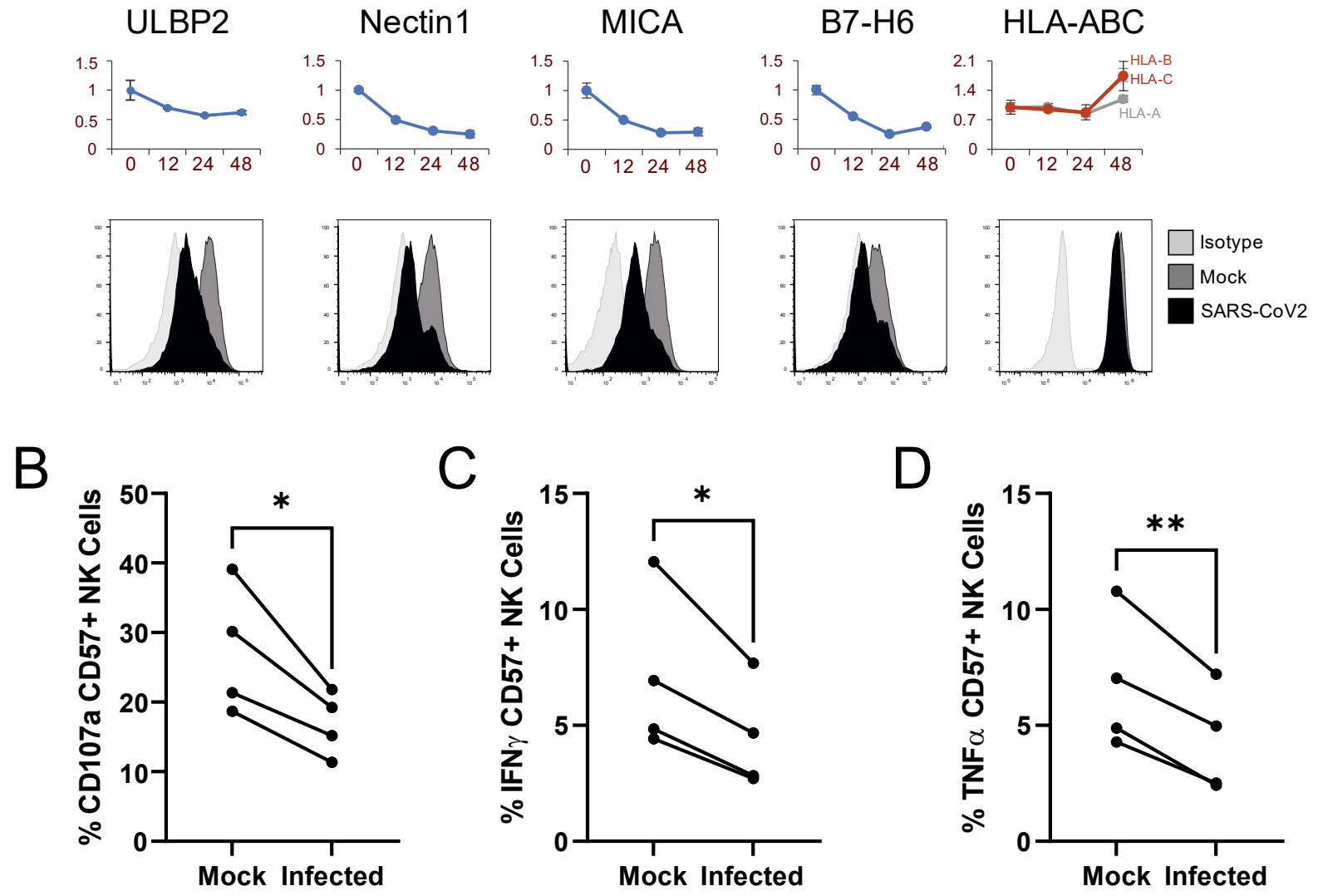

Figure 3. SARS-CoV2 infection leads to downregulation of multiple NK ligands, inhibiting NK activation. (A) AAT cells were either mock infected, or infected with SARS-CoV2 for $24 \mathrm{~h}$, detached using TrypLE, then stained for the indicated NK cell receptors before being analysed by flow cytometry (bottom). Plots for the same proteins from PMP are included for reference (top) (B) AAT cells were either mock infected, or infected with SARS-CoV2 for $24 \mathrm{~h}$, detached with TrypLE and mixed with PBMC for $5 \mathrm{~h}$ in the presence of golgistop, golgiplug, and CD107a antibody, before staining for CD3/CD56/CD57 and Live/Dead Aqua. Cells were then fixed, permeabilised, and stained for TNF $\alpha$ and IFN $\gamma$. Cells were gated on live CD57+ NK cells, and the percentage of cells positive for CD107a, TNF $\alpha$ and IFN $\gamma$ calculated. Individual assays were run in triplicate, with data combined from multiple donors as indicated. Kruskal-Wallis, ${ }^{*} p<0.05, * * p<0.01$. 

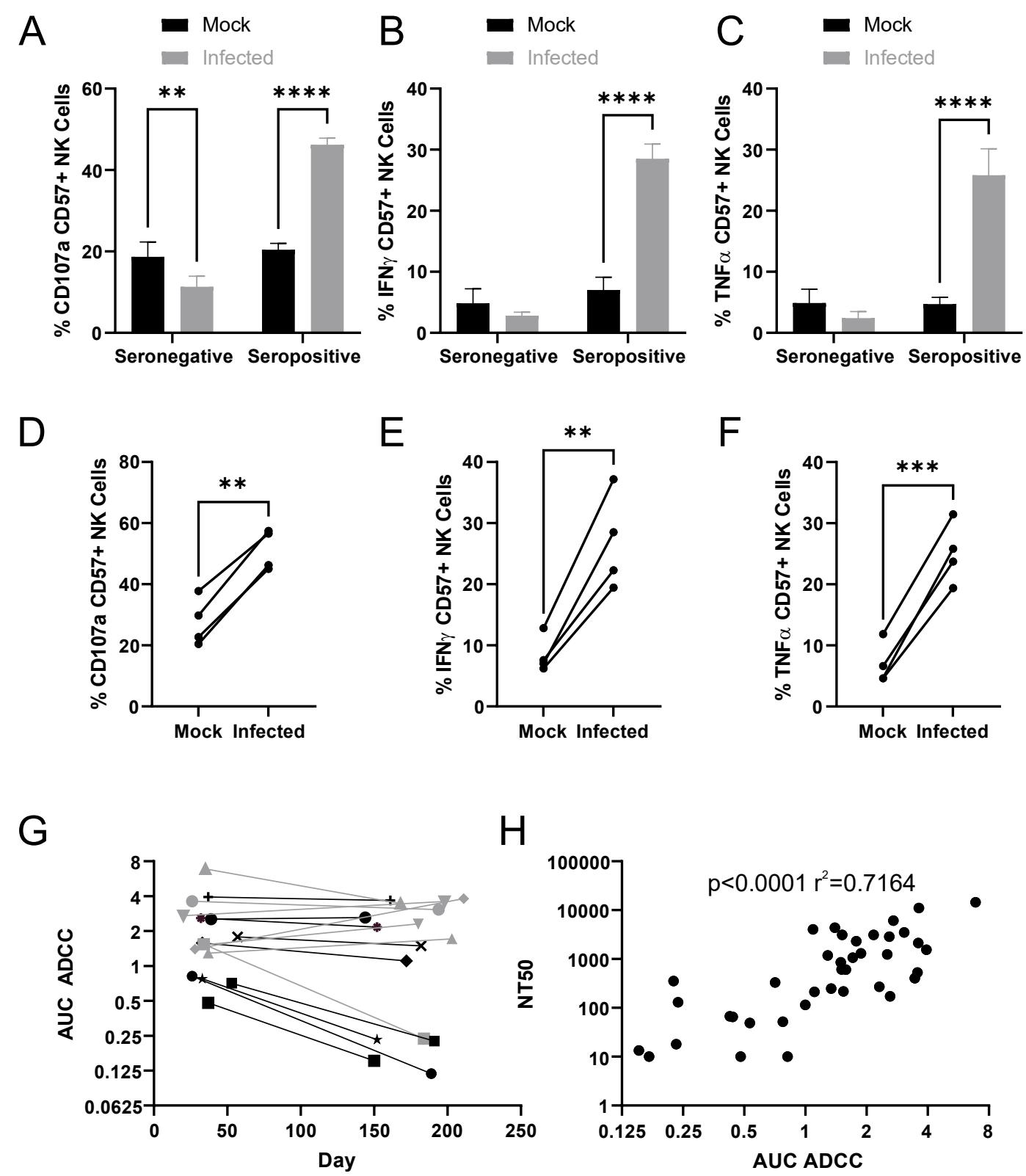

Figure 4. SARS-CoV2 inhibition of NK activation can be overcome via ADNKA. AAT cells were either mock infected, or infected with SARS-CoV2 for 24h, detached using TrypLE, then mixed with PBMC in the presence of golgistop, CD107a antibody, and serum from donors who were seronegative or seropositive for SARS-CoV2. After 5h, cells were stained for CD3, CD56, CD57, and live/dead aqua, then analysed by flow cytometry. (A-F) assays were performed using $1 \%$ serum, and the percentage of CD57+ NK cells positive for CD107a (A, D), TNF $\alpha(B, E)$, and IFN $\gamma$ (C, F) were calculated. Individual assays were performed in triplicate (A-C), 2-way ANOVA $* * p<0.01, * * * * p<0.0001$. Assays from multiple donors were also compared (D-E), KruskalWallis $* * p<0.01, * * * p<0.001$. (G-H) assays were performed using a serial 3-fold dilution of serum, starting from 3.3\%, then the area under the curve (AUC) calculated. (G) longitudinal serums from donors who experienced mild (black) or severe (grey) were used. (H) In addition to AUC for ADCC activity, serums were tested for their ability to neutralise SARS-COV2 infection of VeroE6 cells, and the NT50 calculated, then compared to the AUC for ADCC; Spearman rank correlation analysis is shown. 
A

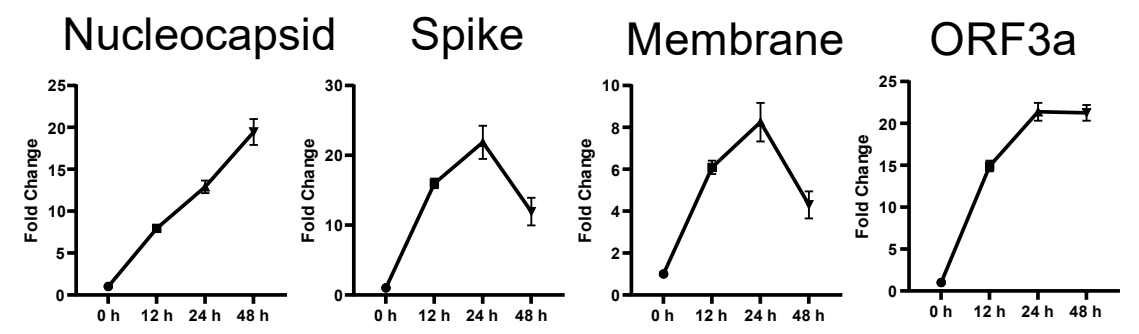

B

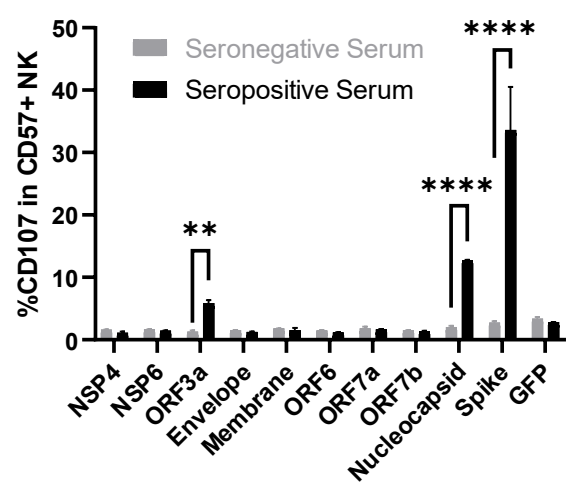

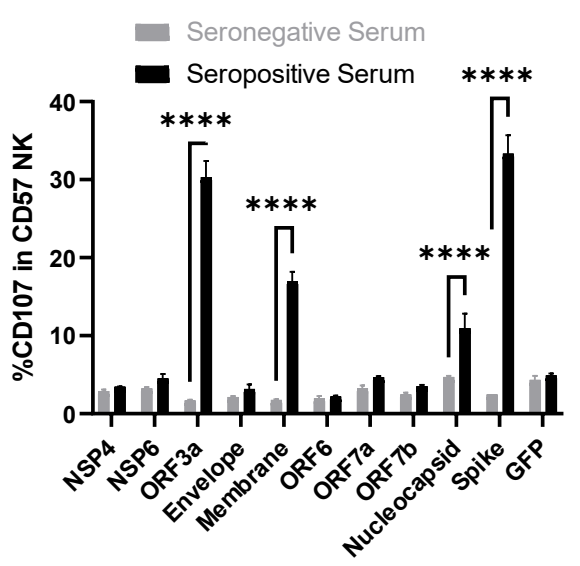

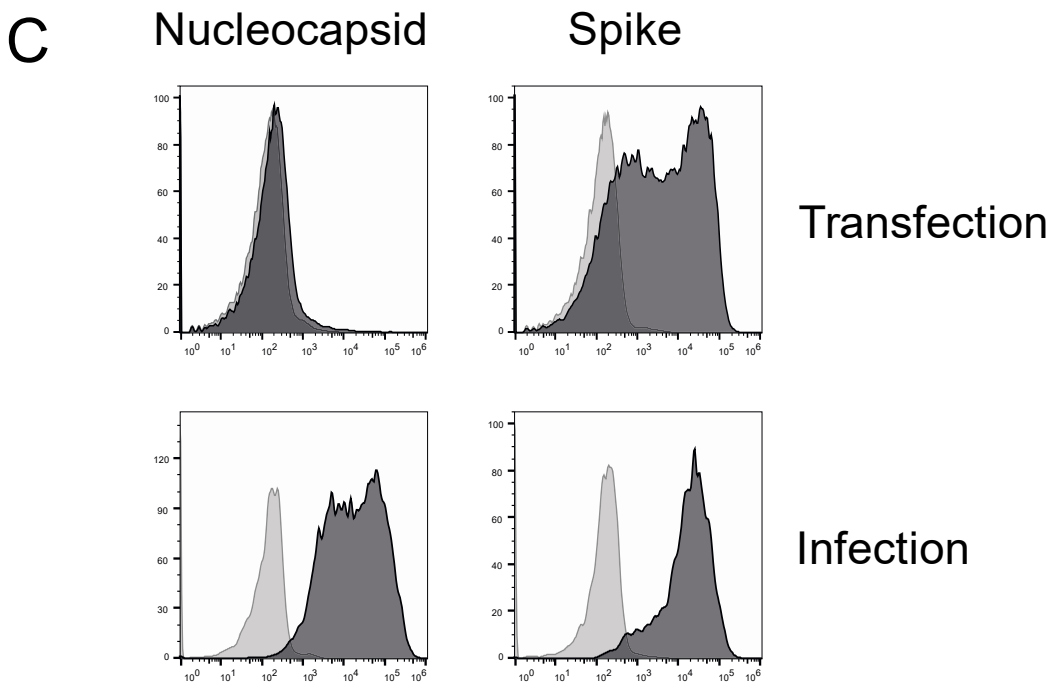

Figure 5. Multiple SARS-CoV2 proteins mediate ADCC. (A) Plots of viral proteins detected in the PMP analysis. (B) 293T cells were transfected with plasmids expressing the indicated SARS-CoV2 proteins for $48 \mathrm{~h}$, then mixed with PBMC in the presence of golgistop, CD107a antibody, and $1 \%$ serum from donors who were seronegative or seropositive for SARS-CoV2. After $5 \mathrm{~h}$, cells were stained for CD3, CD56, CD57, and live/dead aqua, then analysed by flow cytometry. Assays were performed in triplicate. Data is shown from two donor serums. $* * p<0.01, * * * * p<0.0001,2$-way ANOVA. (C) 293 T cells were transfected with plasmid expression SARS-CoV2 nucleocapsid for $48 \mathrm{~h}$, or AAT cells were infected with SARS-CoV2 for 24h, then cells were detached with TrypLE, stained for nucleocapsid, and analysed by flow cytometry. 

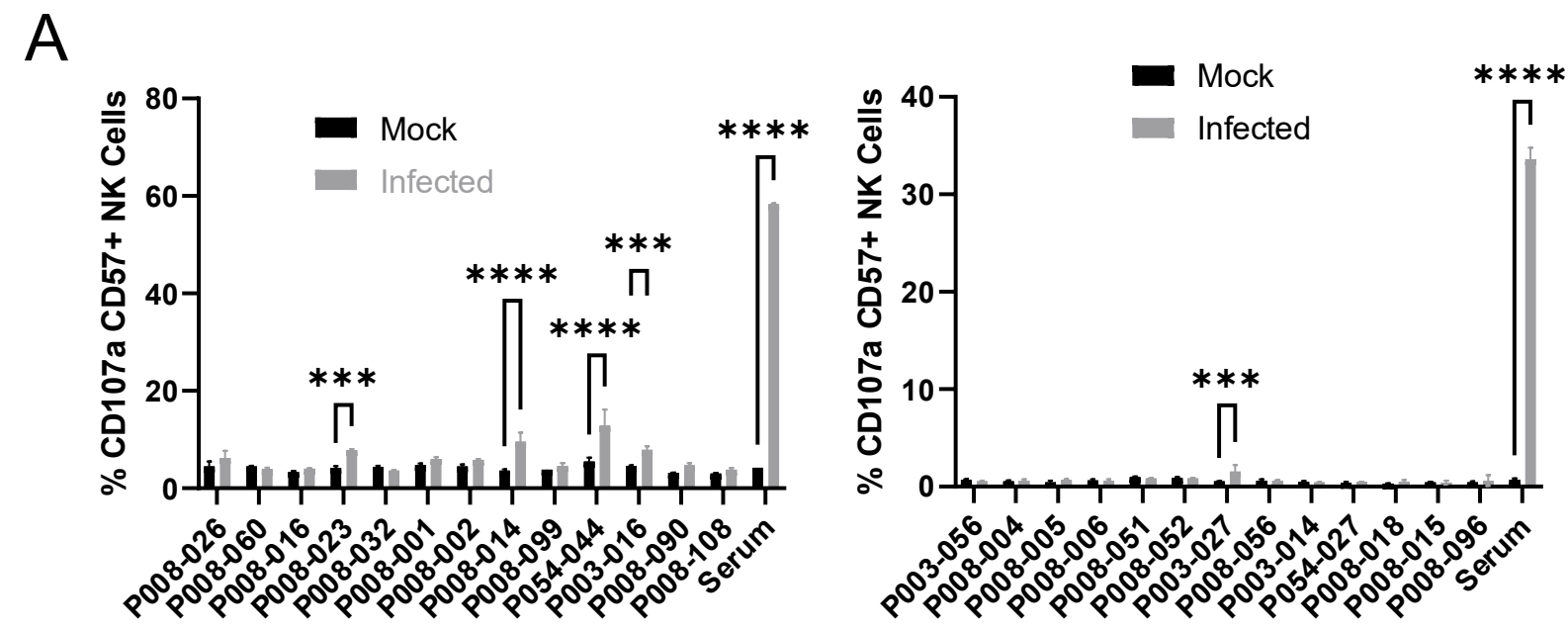

B
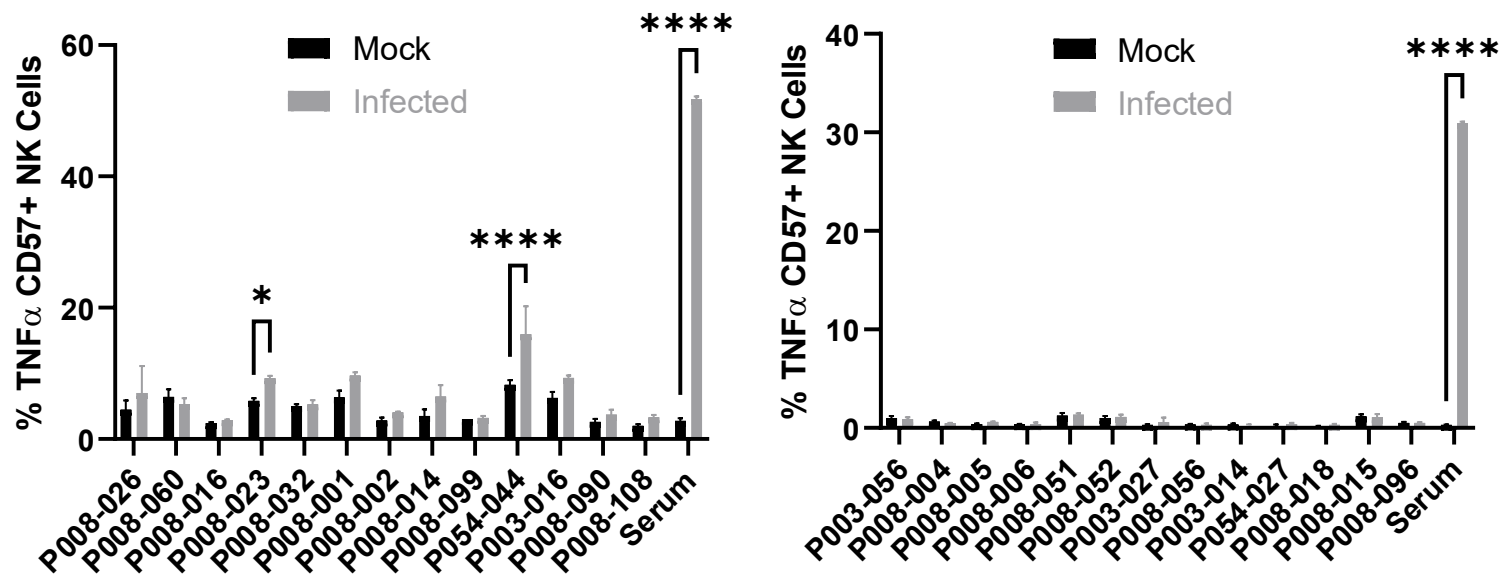

C
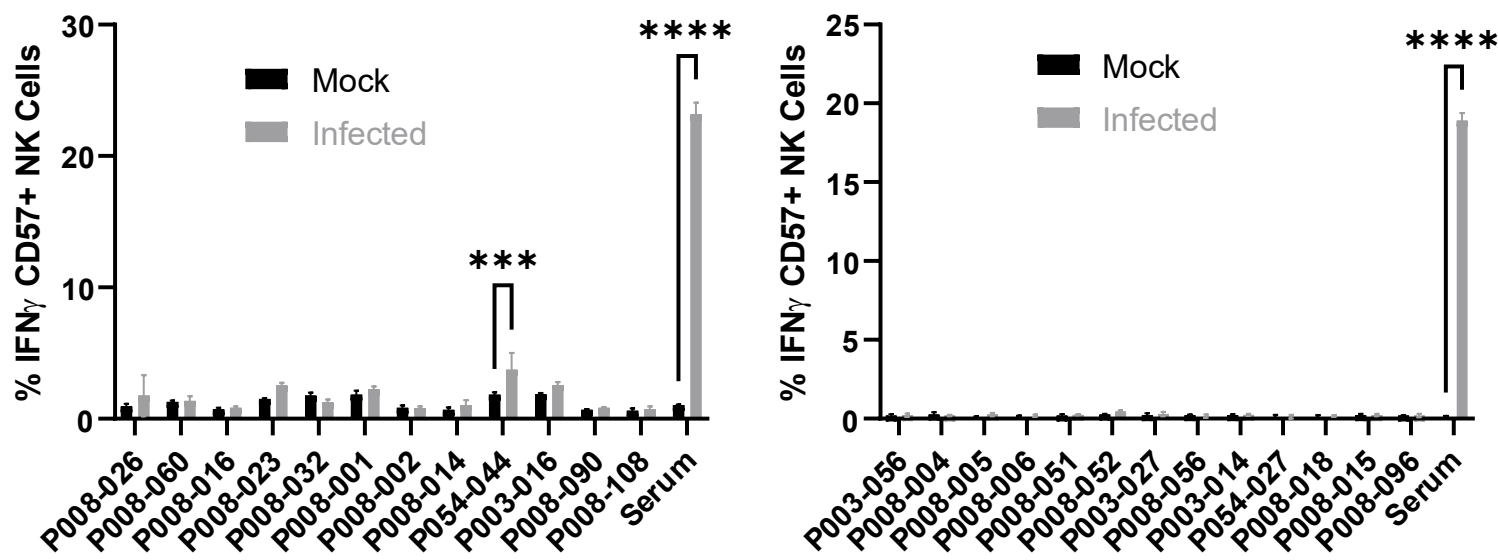

Figure 6. Monoclonal anti-spike antibodies bind infected cells strongly, but only weakly activate ADNKA. AAT cells were either mock infected, or infected with SARS-CoV2 for $24 \mathrm{~h}$, detached with TrypLE and mixed with PBMC for $5 \mathrm{~h}$ in the presence of golgistop, golgiplug, and CD107a antibody, before staining for CD3, CD56, CD57 and Live/Dead Aqua. Cells were then fixed, permeabilised, and stained for TNF $\alpha$ and IFN $\gamma$. Cells were gated on live CD57+ NK cells, and the percentage of cells positive for CD107a (A), TNF $\alpha$ (B) and IFN $\gamma$ (C) calculated. Assays were run in triplicate. 2-way ANOVA ***p<0.001, ****p<0.0001. 
Natural Infection

A
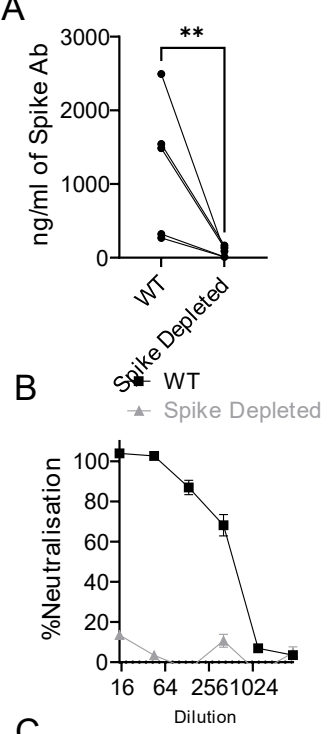

C

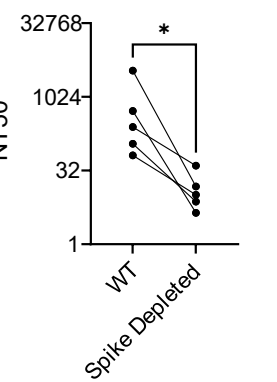

D $\quad-W T$

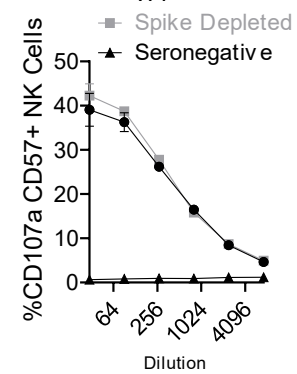

$E$

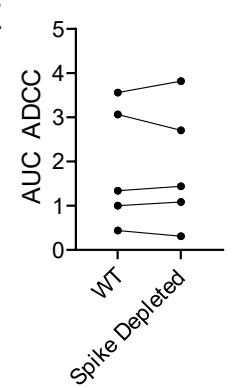

$\underline{\text { Vaccination }}$

$\mathrm{F}$

G

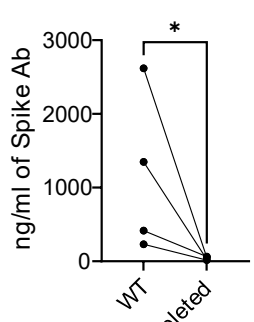

$\mathrm{H}$

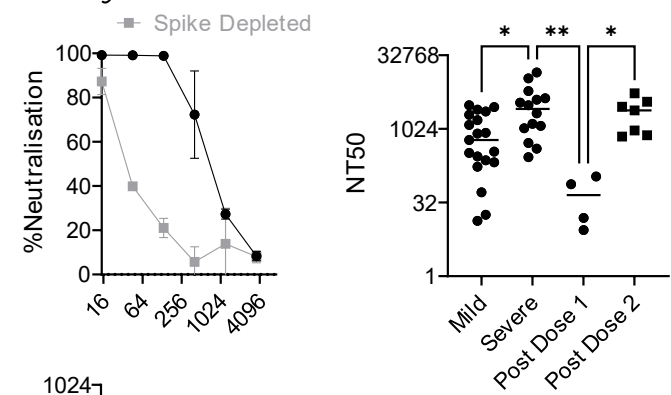

I
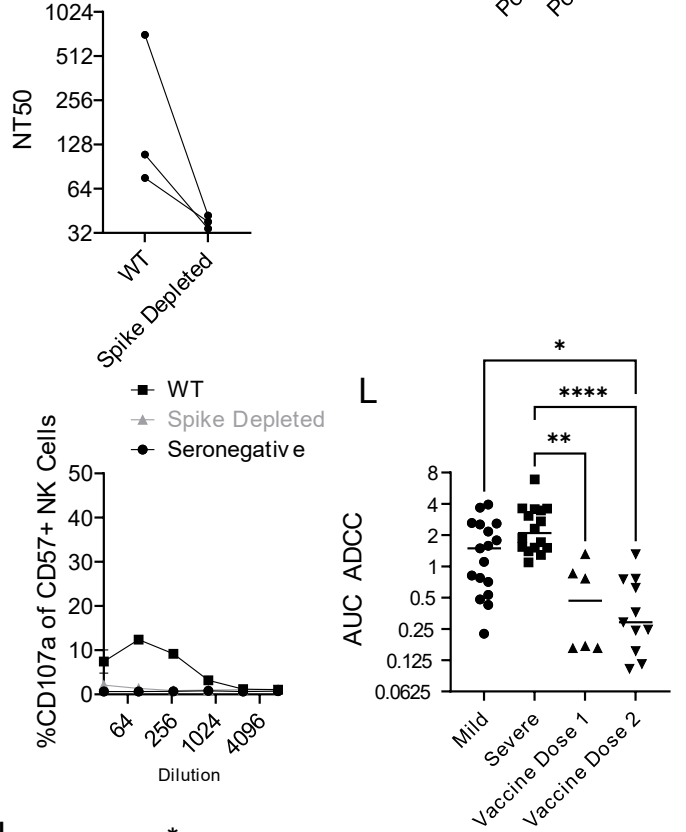

Figure 7. Antibodies targeting spike are weak activators of ADNKA, and the ADNKA response is dominated by non-spike antibodies following natural infection. Serums from individuals naturally infected with SARS-CoV2 (A-E), or donors that had been vaccinated against SARSCoV2 but were seronegative prior to vaccination (F-J) were depleted of anti-spike antibodies using spike Trimer protein conjugated to magnetic beads. ( $A, F)$ ELISA for spike trimer was 
used to measure levels of antibodies before and after depletion. $(B, C, G, H)$ the ability of the original, or anti-spike depleted, serums to neutralise the ability of SARS-CoV2 to infect VeroE6 cells was determined across a range of concentrations, then NT50 values calculated. Example plots $(B, G)$ and NT50 values for multiple donors $(C, H)$ are shown. $(D, E, I, J)$ AAT cells were either mock infected, or infected with SARS-CoV2 for $24 \mathrm{~h}$, detached using TrypLE, then mixed with PBMC in the presence of golgistop, CD107a antibody, and serial dilutions of serum. After $5 \mathrm{~h}$, cells were stained for CD3, CD56, CD57, and live/dead aqua, then analysed by flow cytometry for the percentage of CD107a positive CD57+ NK cells. AUC values were then calculated. Example plots $(D, I)$ and AUC values for multiple donors (E, J) are shown. NT50 (K) and $A \cup C(L)$ values are also given for samples categorised according to disease or vaccination status. Kruskal-Wallis $* p<0.05, * * p<0.001$. 
A

B
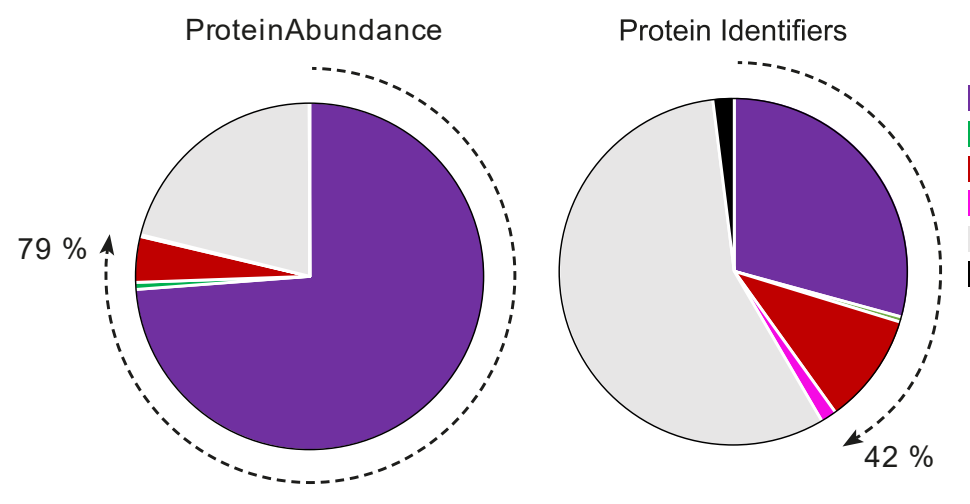

Plasma membrane

Cell Surface

Extracellular

Short Go: membrane

Other

No GO:CC

Inteferon Alpha Response

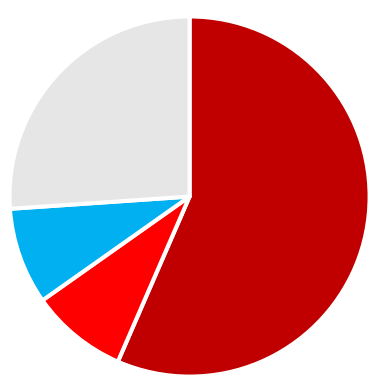

Cluster 5

Cluster 3

Cluster 1

Not clustered

C

Cluster 5

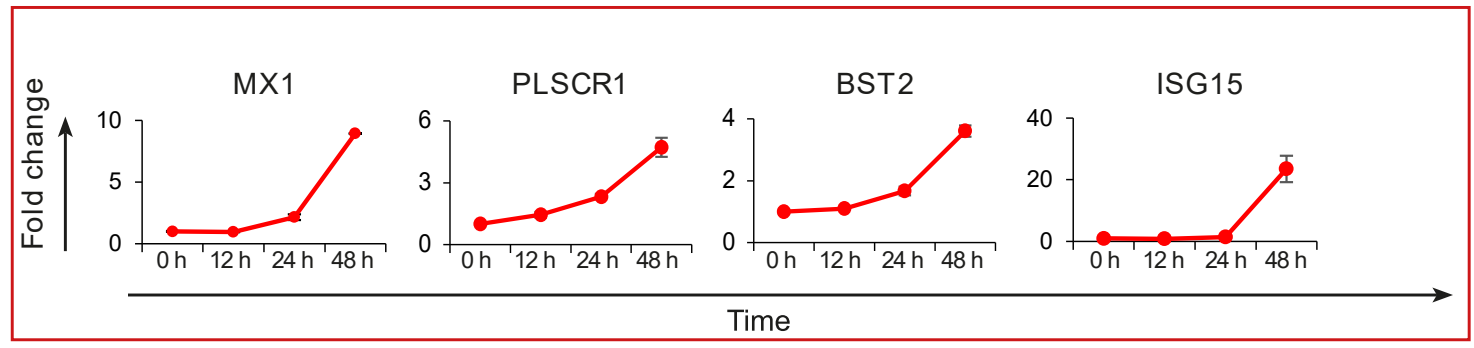

Figure S1. Plasma Membrane Proteins are enriched in the PMP dataset. (A) Pie charts showing the proportion of proteins falling into previously defined annotation categories for plasma membrane proteins by left, protein abundance, or right, protein identifiers. (B) Pie chart showing the distribution of proteins defined by the Molecular Signatures Database (MSigDB) gene set "Hallmark Interferon Alpha Response" into the defined temporal clusters. C. Example temporal profiles of type I interferon inducible genes upregulated in cluster 5, yaxis shows fold change compared to $0 \mathrm{~h}$ timepoint. 


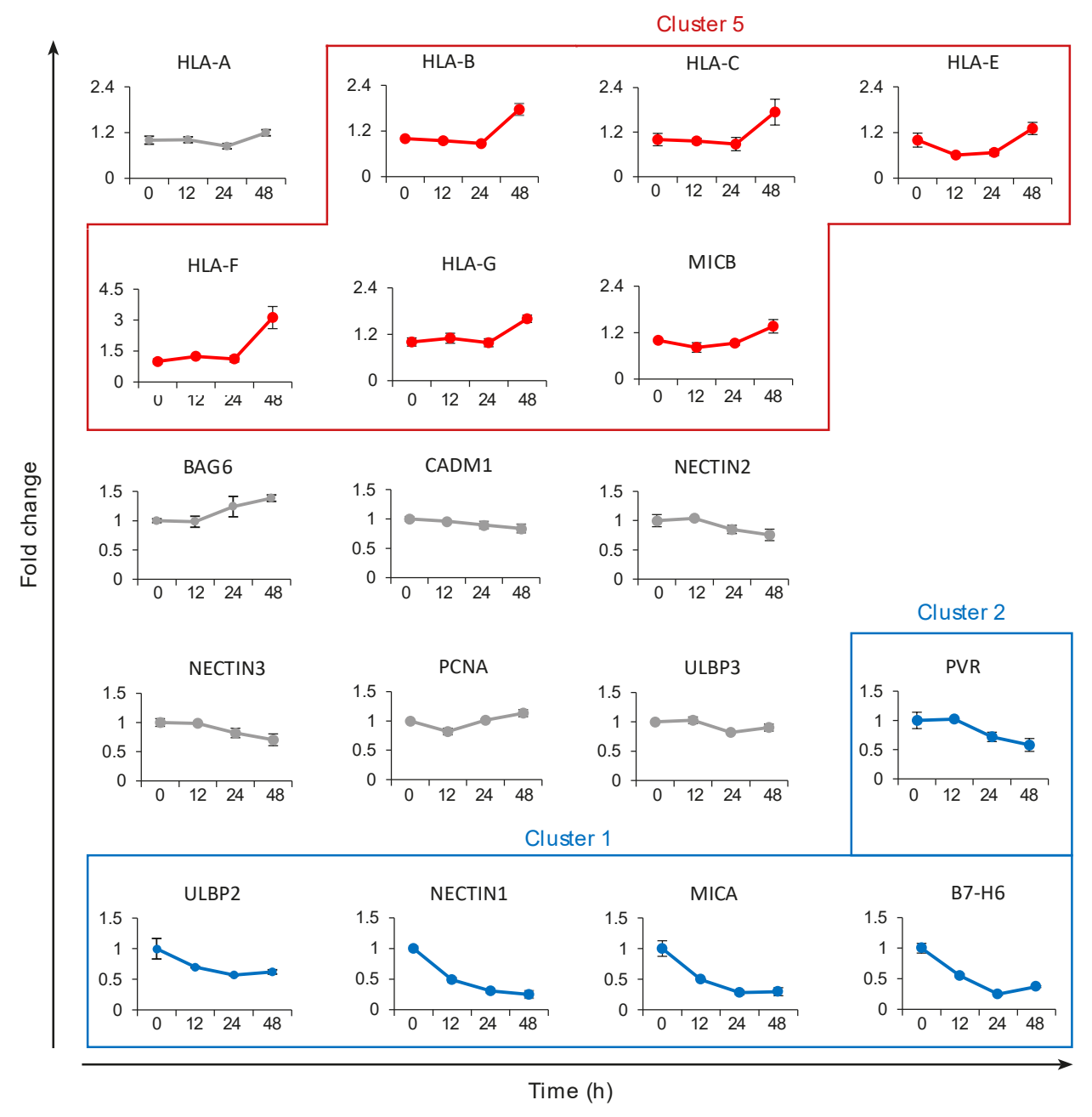

Figure S2. NK ligands detected in the PMP Dataset. Temporal profiles of NK-cell ligands detected in the PMP dataset, $\mathrm{y}$-axis shows fold change compared to $0 \mathrm{~h}$ timepoint. 

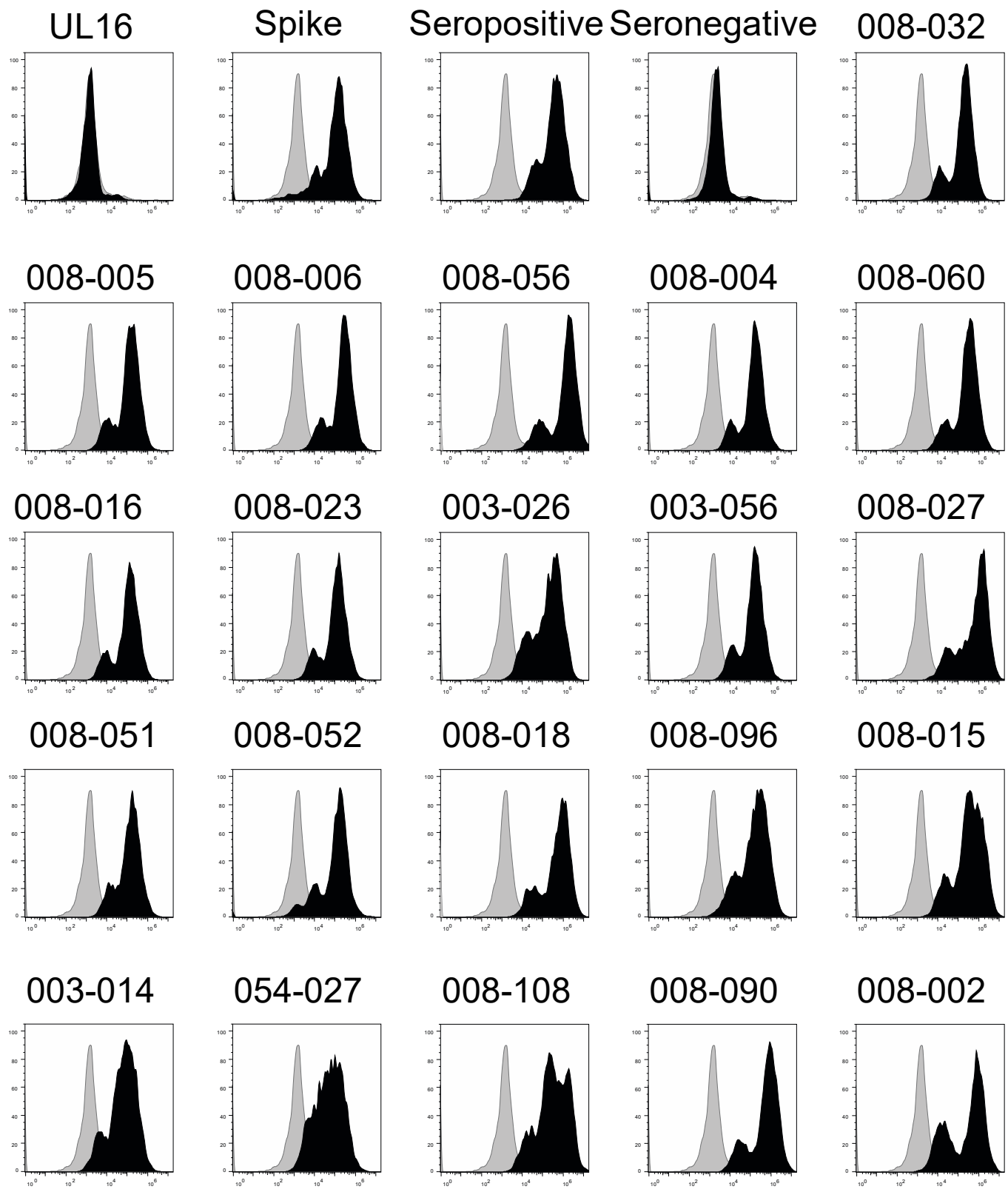

008-015
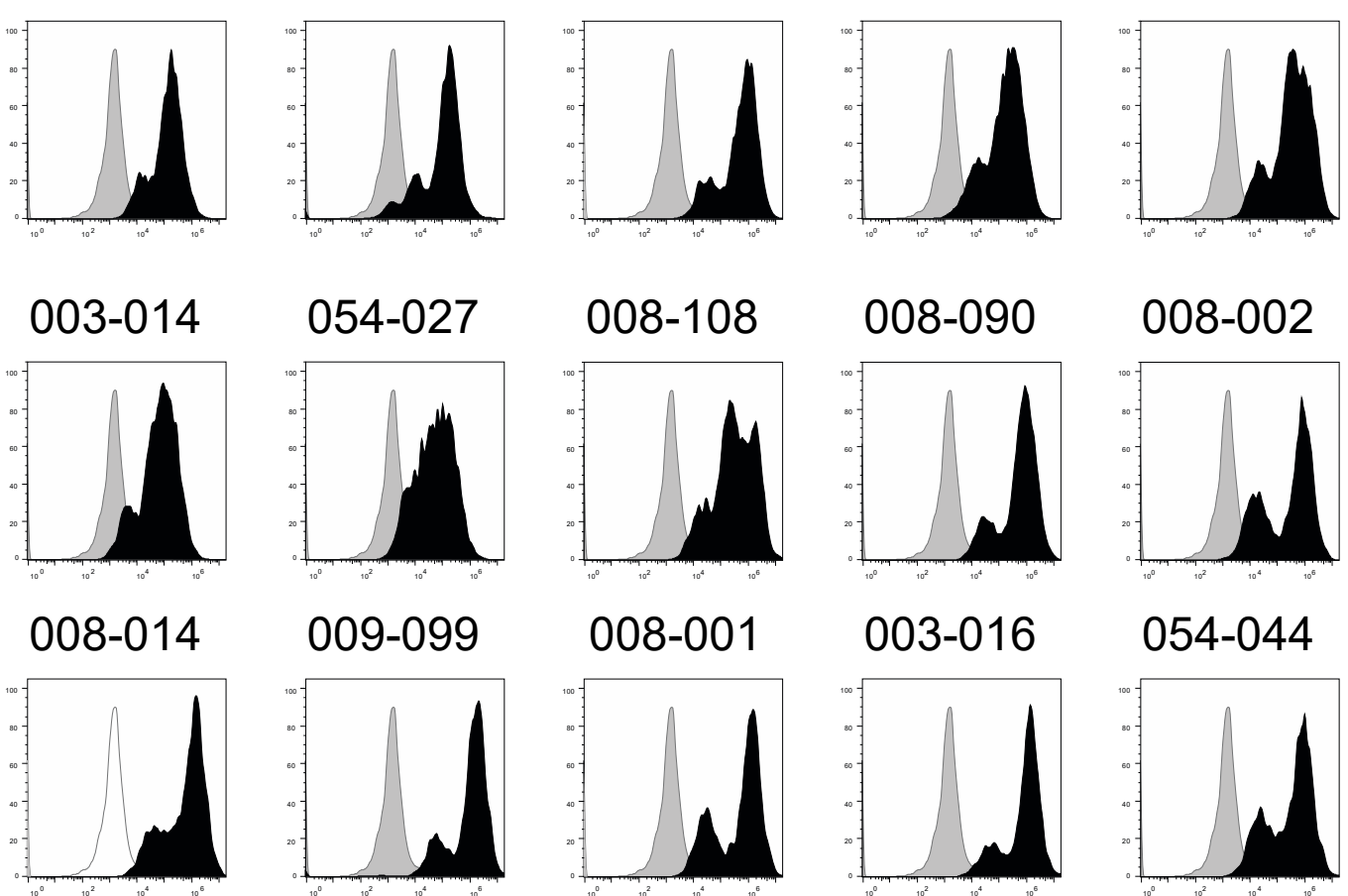

008-002

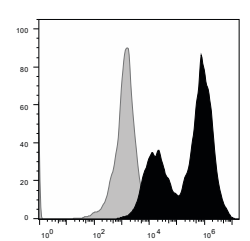

054-044

Figure S3. Anti-spike antibodies bind to SARS-CoV2 infected cells. AAT cells were mock infected (light grey), or infected with SARS-CoV2 (black) for $24 \mathrm{~h}$, then detached with TrypLE, 
bioRxiv preprint doi: https://doi.org/10.1101/2021.04 06.438630; this version posted April 6, 2021. The copyright holder for this preprint (which was not certified by peer review) is the author/funder, who has granted bioRxiv a license to display the preprint in perpetuity. It is made available under aCC-BY-NC-ND 4.0 International license.

stained with the indicated human anti-spike antibodies, then analysed by flow cytometry.

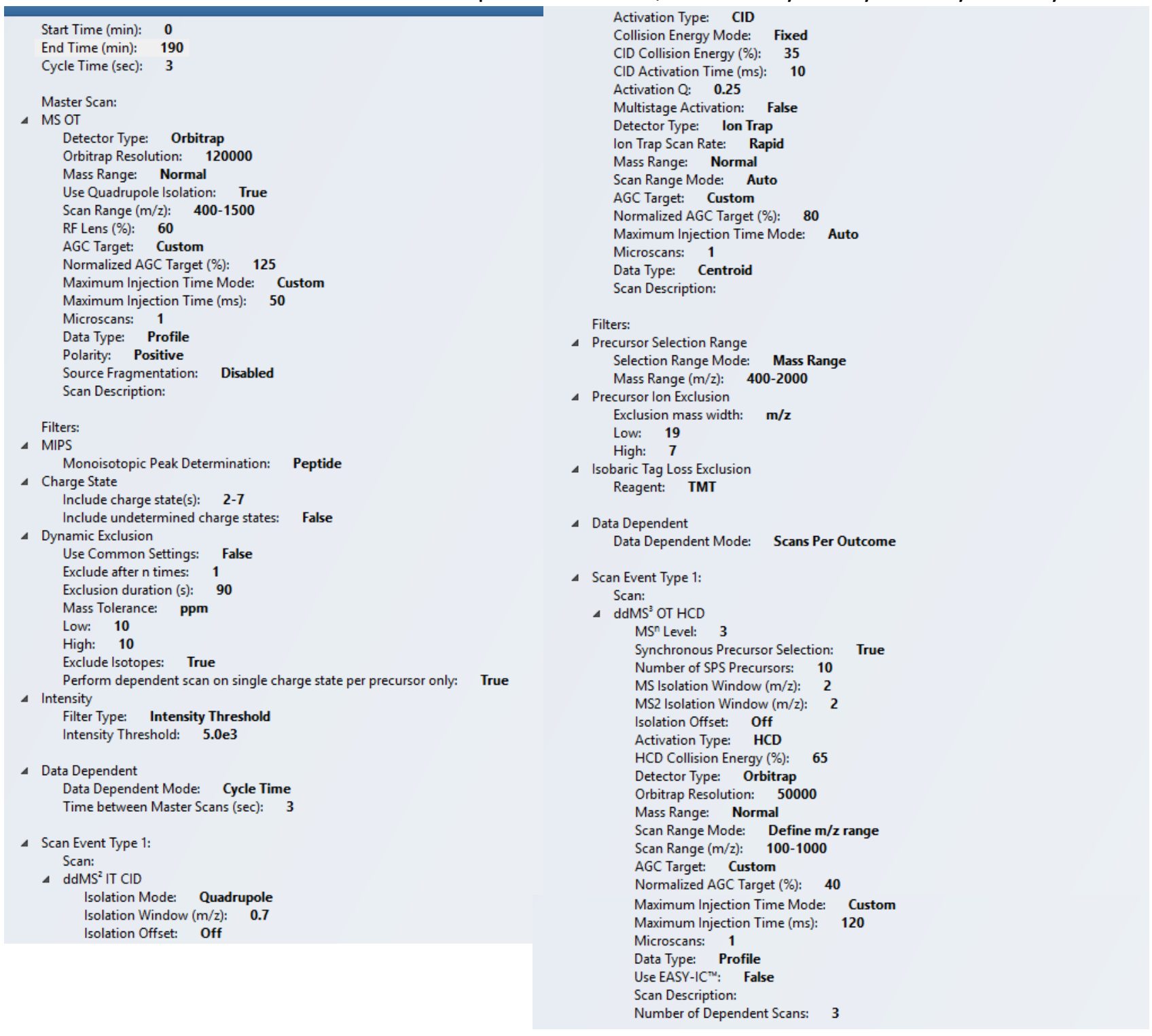

\section{Figure S4. MS Method parameters}




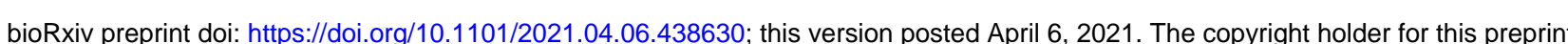
(which was not certified by peer review) is the author/funder, who has granted bioRxiv a license to display the preprint in perpetuity. It is made available under aCC-BY-NC-ND 4.0 International license.

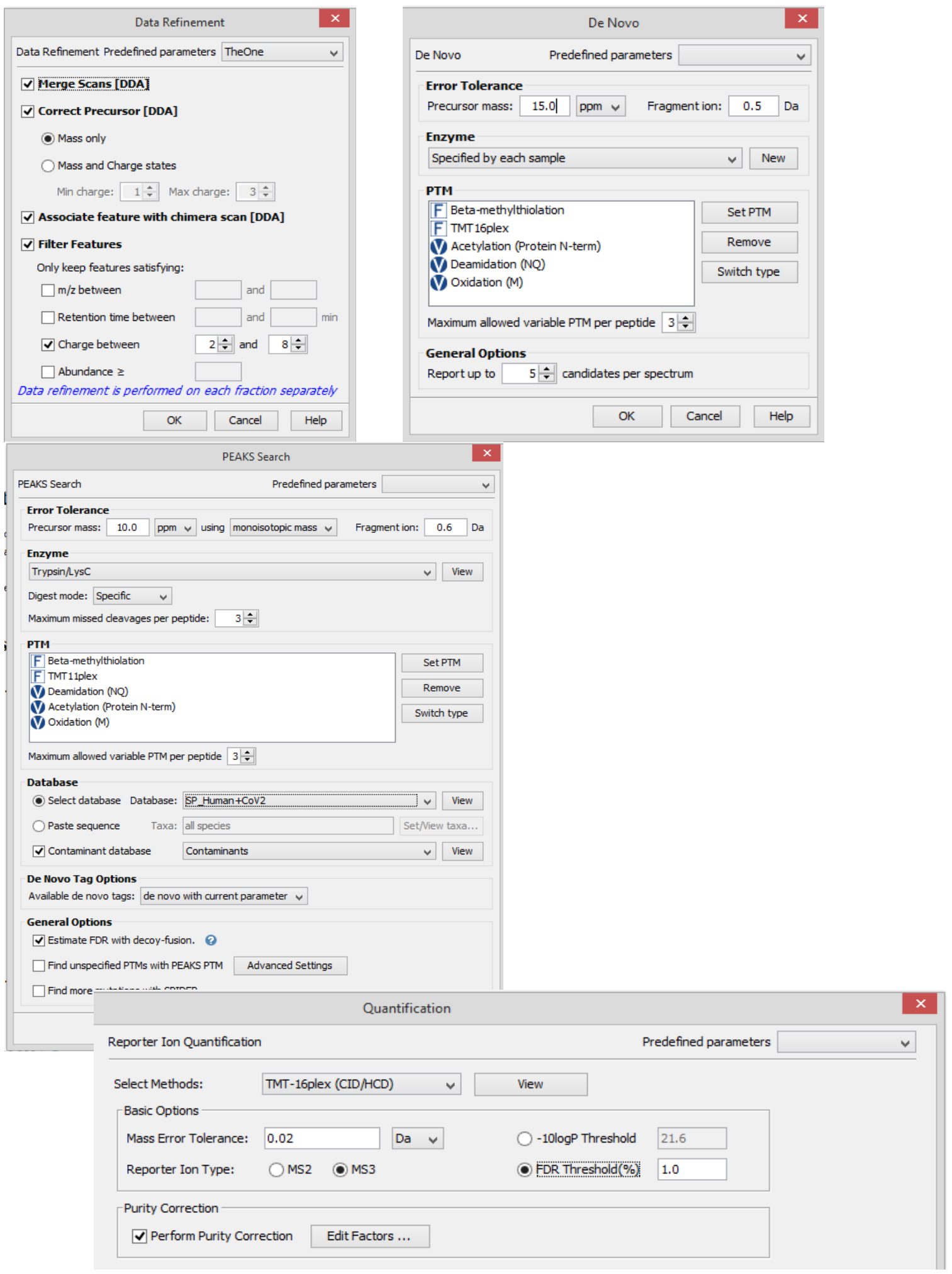

Figure S5. Search Parameters for MS data processing 
Table S2. Clinical characteristics of patients giving longitudinal serum samples

\begin{tabular}{|c|c|c|c|c|}
\hline $\begin{array}{l}\text { Study } \\
\text { ID }\end{array}$ & Severity & Age & Gender & Past medical history \\
\hline 1 & Mild & 82 & M & dementia, ESRF, HTN, IHD, PVD \\
\hline 2 & Mild & 77 & $\mathbf{F}$ & ESRF, HTN, T2DM \\
\hline 3 & Mild & 64 & $\mathbf{F}$ & AF, CVA, dementia, ESRF, HTN, T2DM \\
\hline 4 & Mild & 27 & $\mathbf{F}$ & ESRF, obesity \\
\hline 5 & Mild & 48 & $\mathbf{F}$ & ESRF, HTN, obesity \\
\hline 6 & Severe & 52 & $\mathbf{F}$ & Asthma, obesity \\
\hline 7 & Severe & 62 & M & $\begin{array}{l}\text { colon ca, epilepsy, HTN, obesity, } \\
\text { T2DM }\end{array}$ \\
\hline 8 & Severe & 65 & $\mathbf{F}$ & HTN, hypothyroidism, obesity \\
\hline 9 & Severe & 61 & M & ESRF, obesity, VTE \\
\hline 10 & Severe & 57 & M & \\
\hline 11 & Mild & 23 & $\mathbf{F}$ & CKD, HTN, T1DM \\
\hline 12 & Mild & 62 & M & ESRF, HTN, renal transplant \\
\hline 13 & Mild & 25 & $\mathbf{F}$ & AML \\
\hline 14 & Mild & 52 & M & CVA, prostate ca, T2DM \\
\hline 15 & Mild & 88 & $\mathbf{F}$ & AF, CCF, CKD, COPD, IHD, RA, T2DM \\
\hline 16 & Mild & 64 & $\mathbf{M}$ & \\
\hline 17 & Mild & 69 & M & AF, HTN, T2DM, VTE \\
\hline 18 & Mild & 22 & M & NHL \\
\hline 19 & Severe & 49 & M & \\
\hline 20 & Severe & 67 & $\mathbf{F}$ & AF, HTN, obesity, T2DM \\
\hline
\end{tabular}

$A F=$ atrial fibrillation, $A M L=$ acute myeloid leukaemia, ca=cancer, $C C F=$ congestive cardiac failure, $C K D=$ chronic kidney disease, $C O P D=$ chronic obstructive pulmonary disease, CVA cerebrovascular accident, ESRF=end-stage renal failure, HTN=hypertension, IHD=ischaemic heart disease, $\mathrm{NHL}=$ non-Hodgkin lymphoma, RA=rheumatoid arthritis, T1DM/T2DM=type $1 / 2$ diabetes mellitus, VTE=venous thromboembolism. 


\begin{tabular}{|c|c|c|c|c|c|c|c|c|}
\hline $\begin{array}{l}\text { mAb } \\
\text { name }\end{array}$ & Specificity & $\begin{array}{c}\text { Competition } \\
\text { group }\end{array}$ & S EC50 & $\begin{array}{l}\text { RBD } \\
\text { EC50 }\end{array}$ & $\begin{array}{l}\text { NTD } \\
\text { EC50 }\end{array}$ & $\begin{array}{c}\text { S2 } \\
\text { ELISA }\end{array}$ & $\begin{array}{l}\text { SARS- } \\
\text { COV-2 } \\
\text { WT FL } \\
\text { IC50 }\end{array}$ & $\begin{array}{l}\text { SARS- } \\
\text { CoV-2 } \\
\text { WT PV } \\
\text { IC50 }\end{array}$ \\
\hline P008_060 & NON-S1 & 7 & 0.031 & $>10$ & $>10$ & n.d. & 32.90 & 0.095 \\
\hline P008_004 & NON-S1 & n.d. & 0.045 & $>10$ & $>10$ & + & $>100$ & $>100$ \\
\hline P008_005 & NON-S1 & n.d. & 0.20 & $>10$ & $>10$ & + & $>100$ & $>100$ \\
\hline P008_006 & NON-S1 & n.d. & 0.10 & $>10$ & $>10$ & + & $>100$ & $>100$ \\
\hline P008_016 & NON-S1 & n.d. & 0.11 & $>10$ & $>10$ & + & $>100$ & $>100$ \\
\hline P008_023 & NON-S1 & n.d. & 0.40 & $>10$ & $>10$ & + & $>100$ & $>100$ \\
\hline P008_032 & NON-S1 & n.d. & 0.060 & $>10$ & $>10$ & + & $>100$ & $>100$ \\
\hline P008_051 & NTD & 5 & 0.091 & $>10$ & 0.056 & n.d. & 48.82 & 0.30 \\
\hline P008_052 & NTD & 5 & 0.085 & $>10$ & 0.063 & n.d. & 48.65 & 0.13 \\
\hline P003_027 & NTD & 6 & 0.0039 & $>10$ & 0.0065 & n.d. & 1.64 & 12.92 \\
\hline P008_056 & NTD & 6 & 0.060 & $>10$ & 0.049 & n.d. & 0.014 & $>100$ \\
\hline P008_001 & NTD & n.d. & 0.82 & $>20$ & 1.20 & n.d. & $>100$ & $>100$ \\
\hline P008_002 & NTD & n.d. & 0.0084 & $>20$ & 0.015 & n.d. & $>100$ & $>100$ \\
\hline P008_014 & NTD & n.d. & 0.066 & $>20$ & 0.021 & n.d. & 1.81 & $>100$ \\
\hline P008_099 & NTD & n.d. & 0.022 & $>20$ & 0.15 & n.d. & $>100$ & $>100$ \\
\hline P054_044 & NTD & n.d. & 0.031 & $>20$ & 4.00 & n.d. & 0.94 & 0.74 \\
\hline P003_016 & NTD & n.d. & 0.13 & $>20$ & 2.00 & n.d. & 0.15 & 0.45 \\
\hline P003_014 & RBD & 1 & 0.0076 & 0.0086 & n.d. & n.d. & $>100$ & $>100$ \\
\hline P054_027 & RBD & 1 & 0.0077 & 0.013 & n.d. & n.d. & $>100$ & 0.7 \\
\hline P008_018 & RBD & 3 & 0.071 & 0.082 & n.d. & n.d. & 0.055 & 0.00081 \\
\hline P008_090 & RBD & 3 & 0.018 & 0.026 & n.d. & n.d. & 0.071 & 0.0042 \\
\hline P008_108 & RBD & 3 & 0.0092 & 0.014 & n.d. & n.d. & 0.0023 & 0.0031 \\
\hline P008_015 & RBD & 4 & 0.034 & 0.036 & n.d. & n.d. & 0.055 & 0.00012 \\
\hline P008_096 & RBD & 4 & 0.030 & 0.055 & n.d. & n.d. & 9.09 & 0.41 \\
\hline
\end{tabular}

Table S3. Monoclonal Ant-spike Antibodies used, data taken from ${ }^{44}$ 